\title{
Haemoglobin scavenging in intracranial bleeding: biology and clinical implications
}

Diederik Bulters ${ }^{1,10}$, Ben Gaastra, ${ }^{10}$, Ardalan Zolnourian', Sheila Alexander ${ }^{2}$ Dianxu Ren², Spiros L. Blackburn ${ }^{3}$, Mark Borsody4, Sylvain Doré ${ }^{5}$, James Galea ${ }^{6}$, Koji lihara ${ }^{7}$, Paul Nyquist ${ }^{8}$ and Ian Galea ${ }^{9 *}$

Abstract | Haemoglobin is released into the CNS during the breakdown of red blood cells after intracranial bleeding. Extracellular free haemoglobin is directly neurotoxic. Haemoglobin scavenging mechanisms clear haemoglobin and reduce toxicity; these mechanisms include erythrophagocytosis, haptoglobin binding of haemoglobin, haemopexin binding of haem and haem oxygenase breakdown of haem. However, the capacity of these mechanisms is limited in the CNS, and they easily become overwhelmed. Targeting of haemoglobin toxicity and scavenging is, therefore, a rational therapeutic strategy. In this Review, we summarize the neurotoxic mechanisms of extracellular haemoglobin and the peculiarities of haemoglobin scavenging pathways in the brain. Evidence for a role of haemoglobin toxicity in neurological disorders is discussed, with a focus on subarachnoid haemorrhage and intracerebral haemorrhage, and emerging treatment strategies based on the molecular pathways involved are considered. By focusing on a fundamental biological commonality between diverse neurological conditions, we aim to encourage the application of knowledge of haemoglobin toxicity and scavenging across various conditions. We also hope that the principles highlighted will stimulate research to explore the potential of the pathways discussed. Finally, we present a consensus opinion on the research priorities that will help to bring about clinical benefits.

Porphyrin ring A complex aromatic chemical structure consisting of four modified pyrrole rings with the capacity to coordinate a central metal ion;

protoporphyrin IX is the porphyrin in haemoglobin.
*e-mail:I.Galea@soton.ac.uk https://doi.org/10.1038/ s41582-018-0020-0
Degradation of red blood cells (RBCs) after haemorrhage and intravascular or extravascular haemolysis results in extracellular release of haemoglobin. Within the CNS, cell-free haemoglobin and its breakdown products are neurotoxic and cause secondary brain injury ${ }^{1,2}$. Two scenarios can lead to haemoglobin coming into direct contact with brain parenchyma: vascular rupture (as occurs during various forms of intracranial haemorrhage) and low-grade leakage of haemoglobin into the brain. Haemoglobin-induced neurotoxicity is thought to have an important role in the pathophysiology of several neurological conditions. These conditions were traditionally thought to be those characterized by acute macroscopic bleeding, such as intracranial haemorrhage, but evidence suggests that extracellular haemoglobin also plays an important role in several neurological conditions, including superficial siderosis ${ }^{3}$, Alzheimer disease ${ }^{4}$ and progressive multiple sclerosis ${ }^{5}$.

In this Review, we consider the emerging importance of extracellular haemoglobin in the brain and its clinical implications. First, we present the cellular and molecular mechanisms of haemoglobin neurotoxicity and clearance, focusing on the special environment of the CNS. Subsequently, we focus on the clinical implications of extracellular haemoglobin within the CNS, reviewing the evidence that links the pathophysiology of cell-free haemoglobin to the clinical presentations and outcomes of several neurological conditions. Finally, we discuss current and emerging treatment strategies designed to augment the clearance of haemoglobin and/or alleviate its toxicity. We also present the outcomes of a meeting of an expert panel in which a consensus opinion on the key research questions that need to be answered was formulated. We hope that the principles highlighted will encourage research into the effects of extracellular haemoglobin in the brain and exploration of the potential for clinical benefits.

\section{Neurotoxicity of haemoglobin}

Haemoglobin consists of four globin chains tightly associated with a haem group, which itself consists of a porphyrin ring that coordinates an iron atom in the $\mathrm{Fe}^{2+}, \mathrm{Fe}^{3+}$ or $\mathrm{Fe}^{4+}$ oxidative state. When outside RBCs, haemoglobin 


\section{Key points}

- Extracellular haemoglobin induces cell death, mainly via oxidation and inflammation.

- Mechanisms in the brain that can mitigate haemoglobin toxicity and enable its clearance are easily overwhelmed by extensive haemolysis after intracranial haemorrhage.

- Variation in genes related to haemoglobin binding and metabolism influence outcomes after subarachnoid haemorrhage or intracerebral haemorrhage.

- Treatments that mitigate haemoglobin toxicity and increase clearance might have clinical benefits for patients with intracranial bleeding.

- Research priorities include prospective genetic association studies and an improved basic scientific understanding of the mechanism of haemoglobin toxicity and its clearance from the brain.

\section{Hemichromes}

Haemoglobin molecules that

can no longer react with

oxygen, characterized by the

binding of the distal histidine to

the central Fe atom;

hemichromes can occur

spontaneously and reversibly,

but denaturation of

haemoglobin alters its

quaternary structure,

facilitating the process and

forming irreversible

hemichromes.

Globin-based radical

Reactions between $\mathrm{Fe}^{3+}$ and

peroxides extract an electron

from the protein globin chain

leaving it in the radical state

(with an unpaired electron),

making it highly reactive; this

free radical is commonly on the

phenoxyl group of a tyrosine

residue, and can migrate.

\section{Necroptosis}

A form of regulated cell death,

morphologically akin to

necrosis, triggered by TNF and

regulated by caspase 8 ; it is

dependent on receptor-

interacting protein kinases 1 and 3 . is oxidized to methaemoglobin and dissociates into dimers. Subsequent modification of globin forms hemichromes, and these degrade into haem, which releases iron. The toxicity of haemoglobin is undisputed neurotoxicity is observed in vitro ${ }^{1}$ and after intracerebral injection of haemoglobin in vivo ${ }^{2}$. Neurons seem to be more vulnerable to this toxicity than glia ${ }^{1}$. The toxicity of haemoglobin is multifactorial but mainly seems to be mediated by four factors: oxidation, inflammation, nitric oxide scavenging and oedema.

\section{Oxidation}

Haem mediates oxidation: in its ferrous $\left(\mathrm{Fe}^{2+}\right)$ and ferric $\left(\mathrm{Fe}^{3+}\right)$ states, haem can react with hydrogen peroxide (which is released by neutrophils, for example) or endogenous lipid hydroperoxides (which are formed by lipoxygenases) to form a highly reactive ferryl $\left(\mathrm{Fe}^{4+}\right)$ form. Such reactions of ferric haem also form a globin-based radical ${ }^{6}$. Ferryl haem and globin-based radicals react directly with lipids and proteins to form free radicals, which self-propagate in the presence of molecular oxygen.

Free radicals lead to destructive modification of membranes, lipids, proteins and nucleic acids and their associated machinery, thereby critically altering cellular and organ function ${ }^{7}$. Molecular signatures of this process, including covalently modified proteins ${ }^{8}$ and oxidized lipids ${ }^{9}$, have been detected in the cerebrospinal fluid (CSF) after subarachnoid haemorrhage (SAH). Free haem is thought to be more toxic than haemoglobin, as its lipophilicity enables its intercalation into membranes ${ }^{10}$.

\footnotetext{
Author addresses

${ }^{1}$ Wessex Neurological Centre, University Hospital Southampton, Southampton,

UK. ${ }^{2}$ School of Nursing, University of Pittsburgh, Pittsburgh, PA, USA.

${ }^{3}$ Department of Neurosurgery, University of Texas Health Science Center at Houston,

Houston, TX, USA.

${ }^{4}$ NeuroSpring, Dover, DE, USA.

${ }^{5}$ Department of Anesthesiology, Neurology, Psychiatry, Psychology, Pharmaceutics and

Neuroscience, College of Medicine, Center for Translational Research in

Neurodegenerative Disease, University of Florida, Gainesville, FL, USA.

${ }^{6}$ Brain Injury Research Group, Manchester Academic Health Sciences Centre, University of Manchester, Manchester, UK.

${ }^{7}$ Department of Neurosurgery, Graduate School of Medical Sciences, Kyushu University, Fukuoka, Japan.

${ }^{8}$ Department of Anesthesiology and Critical Care Medicine, Neurology, Neurosurgery and General Internal Medicine, Johns Hopkins University, Baltimore, MD, USA.

${ }^{9}$ Clinical Neurosciences, Clinical and Experimental Sciences, Faculty of Medicine,

University of Southampton, Southampton, UK.

${ }^{10}$ These authors contributed equally: Diederik Bulters and Ben Gaastra.
}

Haem releases Fe, which drives cell death via $\mathrm{Fe}$ dependent lipid oxidation - known as ferroptosis ${ }^{11}-$ which can be suppressed by the lipid reactive oxygen species inhibitor ferrostatin 1, iron chelators and lipophilic antioxidants.

\section{Inflammation}

The pro-inflammatory effects of haemoglobin breakdown products are mediated in several ways. Methaemoglobin ${ }^{12}$ and haem ${ }^{13}$ are ligands of Toll-like receptor 4 (TLR4), which is expressed by microglia and macrophages; activation of TLR4 causes these cells to secrete tumour necrosis factor (TNF), triggering nuclear factor- $\kappa \mathrm{B}$ $(\mathrm{NF}-\mathrm{\kappa B})$ activation, inflammation and necroptosis ${ }^{14}$. Haem can also activate the NLRP3 (NOD-, LRR- and pyrin domain-containing 3) inflammasome, leading to IL-1 $\beta$ synthesis ${ }^{15}$, and can induce IL-1 $\alpha$ production from mixed glia in culture, which in turn causes neuronal death ${ }^{16}$.

\section{Nitric oxide depletion}

Nitric oxide is depleted by haemoglobin in a process that is rapid and irreversible. In this process, reaction of nitric oxide with oxyferrous haemoglobin forms methaemoglobin and nitrate ${ }^{17}$. Nitric oxide is also consumed by oxygen radicals after $\mathrm{SAH}^{18}$; for example, nitric oxide can react with superoxide to form peroxynitrite. Endothelial nitric oxide synthase that is exposed to peroxynitrite does not function normally and produces superoxide instead of nitric oxide ${ }^{19,20}$; this superoxide causes further nitric oxide consumption.

Nitric oxide is a vasodilator produced by endothelial cells, neurons and microglia. It regulates cerebral vascular tone $e^{21}$ and is a potent platelet inhibitor. Several adverse effects of reduced nitric oxide as a result of haemoglobin-mediated depletion have been reported. Evidence suggests that depletion of nitric oxide contributes to microthrombosis ${ }^{22}$ in cerebral vessels in $\mathrm{SAH}$ in humans and animal models, and is associated with poor clinical outcomes ${ }^{23-25}$. Reduced bioavailability of nitric oxide lowers the threshold for cortical spreading depolarization $^{26}$, an electrical phenomenon observed in the presence of high potassium and haemoglobin levels ${ }^{27}$, which leads to spreading ischaemia and neuronal death. Nitric oxide bioavailability at the vasculature is also important in vasospasm; observations of haemoglobininduced vasospasm in the acute phase of preclinical SAH models ${ }^{28-30}$ gave rise to the long-held belief that an absolute cerebral nitric oxide deficiency was the cause, but evidence that nitric oxide metabolism is phasic after SAH has changed this view ${ }^{31-37}$. Nitric oxide depletion has not been studied in intracerebral haemorrhage, despite evidence that a reduction in perihaematomal blood flow is delayed ${ }^{38}$ (as would be expected secondary to nitric oxide depletion) and unresponsive to osmotic therapy ${ }^{39}$ (suggesting that the reduced blood flow is unrelated to high intracranial pressure).

\section{Oedema}

Several lines of evidence show that cerebral oedema is induced by haemoglobin and its breakdown products. Intraparenchymal injection of haemoglobin breakdown products increased the water and sodium 


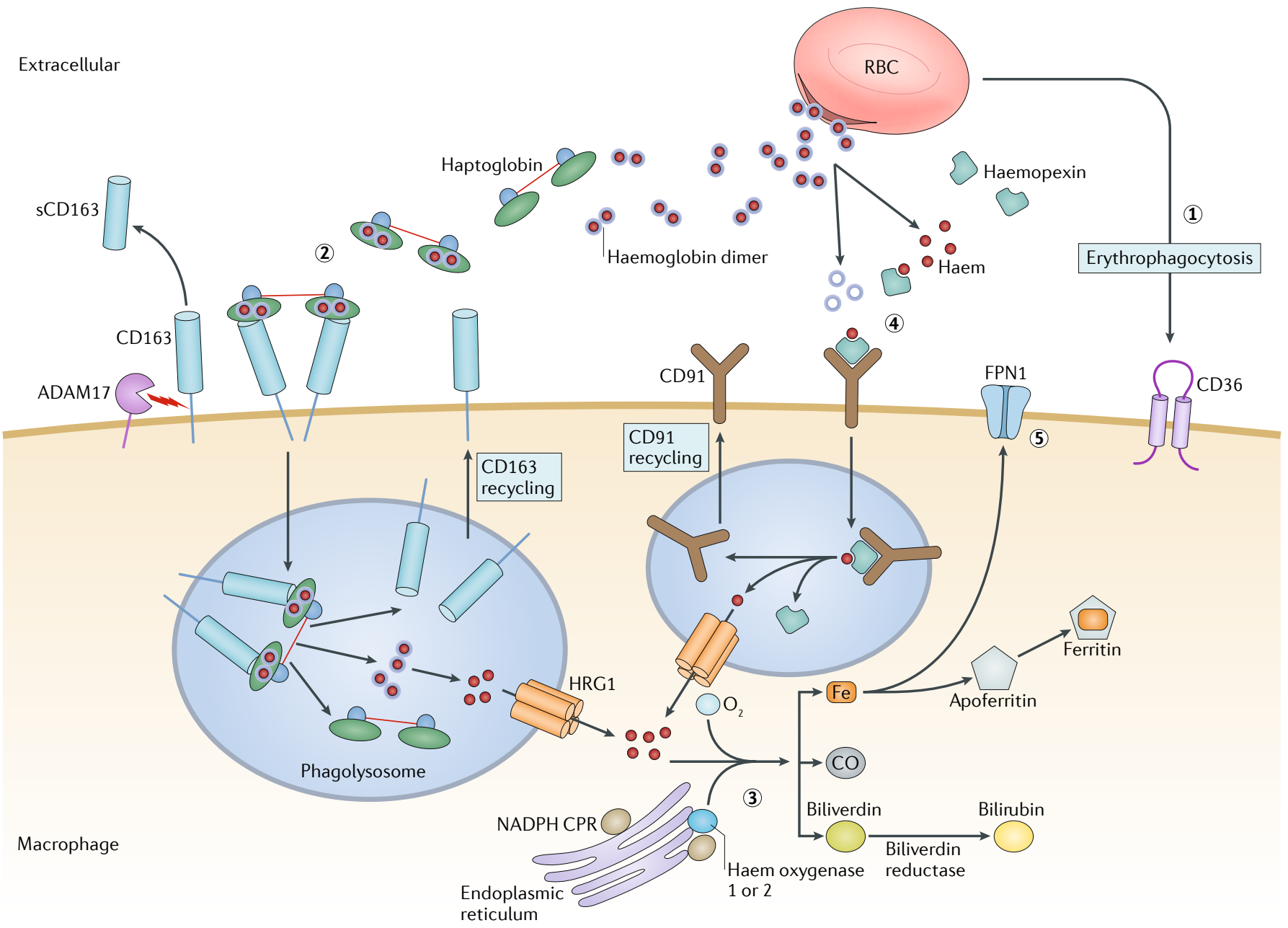

Fig. 1 | Haemoglobin scavenging pathways in humans. Multiple receptor mediated-pathways can prevent the toxicity of haemoglobin and its breakdown products haem and iron. Red blood cells (RBCs) can be directly phagocytosed following detection by the CD36 receptor on macrophages (step 1). Free haemoglobin dimers can be captured by haptoglobin, and the haptoglobin-haemoglobin complex can be taken up by macrophages via CD163 (step 2). CD163 can be shed from the cell surface after subarachnoid haemorrhage owing to the action of the enzyme disintegrin and metalloproteinase domain-containing protein 17 (ADAM17), and this process might reduce the efficiency of haemoglobin scavenging. In the phagolysosome, haemoglobin is then broken down to release haem, which is in turn broken down by haem oxygenases to generate iron (step 3). Similarly, free extracellular haem can be captured by haemopexin and transported into macrophages via CD91 (step 4), where it is also broken down to produce free iron (step 3). The resulting iron is transported from macrophages via the ferroportin 1 (FPN1; also known as SLC40A1) channel (step 5). HRG1, haem-responsive gene 1 protein homologue (also known as SLC48A1); NADPH-CPR, NADPH-cytochrome P450 reductase; $\mathrm{sCD} 163$, soluble CD163.

content in the brains of rats ${ }^{40}$. After haemoglobin injection into the brains of rats, matrix metalloproteinase 9 (MMP-9) was upregulated, resulting in blood-brain barrier (BBB) disruption ${ }^{41}$; acute MMP-9 inhibition attenuated the consequent cerebral oedema ${ }^{42}$. For these reasons, delayed perihaematomal oedema in intracerebral haemorrhage is thought to be at least partly caused by haemoglobin and its breakdown products ${ }^{43}$. Cerebral oedema is more marked in intracerebral haemorrhage than in $\mathrm{SAH}^{44}$, possibly because haemoglobin is in a parenchymal location after intracerebral haemorrhage and is therefore closer to the BBB. In keeping with this speculation, delayed global cerebral oedema is less common after $\mathrm{SAH}^{44}$ and is seen in association with diffuse ischaemia ${ }^{45}$, suggesting a different pathophysiology.

\section{Haemoglobin clearance}

Several endogenous mechanisms have the potential to clear the haemoglobin, haem and iron that are released into the brain following a haemorrhage and RBC lysis (FIG. 1). These mechanisms are discussed below.

\section{Erythrophagocytosis}

Erythrophagocytosis is one mechanism by which abnormal RBCs are cleared. Abnormal RBCs exteriorize phosphatidylserine, and evidence suggests that macrophages recognize phosphatidylserine via CD36, leading to erythrophagocytosis ${ }^{46,47}$. Iron and haem are subsequently exported from the macrophages to the extracellular milieu via, for example, ferroportin ${ }^{48}$ and feline leukaemia virus subgroup C receptor 1 (FLVCR1) ${ }^{49}$, 


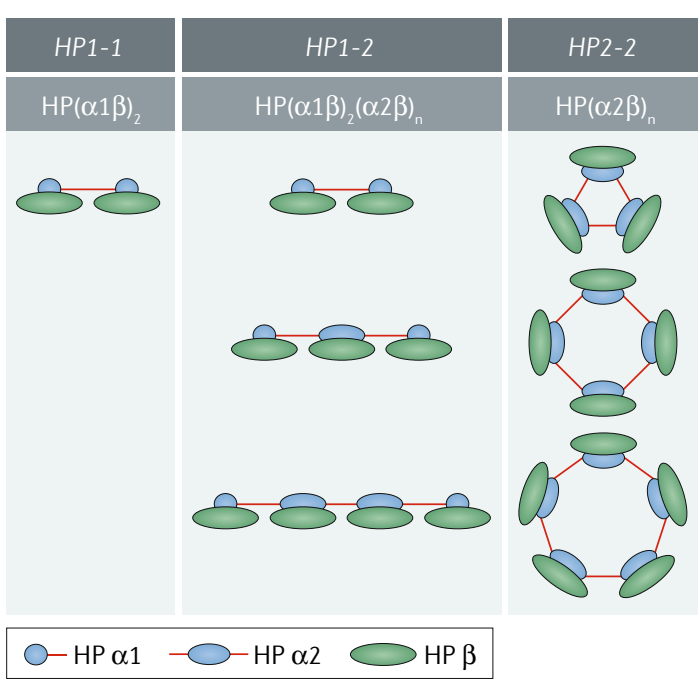

Fig. 2 | Isoforms and possible structures of haptoglobin according to genotype. Haptoglobin is encoded by the HP1 and HP2 alleles. HP1 encodes an a1-chain and a $\beta$-chain that are linked by a disulfide bond. The $\alpha 1$-chain has another free cysteine residue (red) that enables dimerization to form the $\mathrm{HP}(\alpha 1 \beta)_{2}$ dimer (left). This dimer is the only form of haptoglobin present in homozygotes for HP1. HP2 encodes a duplicated $\alpha$-chain called $\alpha 2$ and a $\beta$-chain. The $\alpha 2$-chain has two free cysteines that enable formation of cyclic polymers of increasing size, $\mathrm{HP}(\alpha 2 \beta) n$, where $n \geq 3$ (right). These cyclic polymers are found in homozygotes for HP2. In heterozygotes that express $H P 1$ and $H P 2$, linear polymers form $H P(\alpha 1 \beta)_{2}(\alpha 2 \beta) n$, where $n \geq 0\left(\right.$ REF. $\left.^{218}\right)$.

respectively. Estimates suggest that $40 \%$ of the iron originating from haemoglobin can be released within $24 \mathrm{~h}$ of RBC uptake by macrophages. Although erythrophagocytosis might seem to be an effective clearance mechanism, macrophages that ingest more than two RBCs undergo cell death, leading to release of deleterious haem and iron into the extracellular matrix ${ }^{50}$. Consequently, the contribution of erythrophagocytosis to the prevention of haem-derived toxicity is probably small.

\section{Haptoglobin and CD163}

Extravasated RBCs that avoid erythrophagocytosis undergo spontaneous lysis as a result of, for example, free radical damage or complement-mediated attack ${ }^{51}$, leading to release of haemoglobin. In this situation, the haemoglobin dimer is immediately and irreversibly bound by haptoglobin, an acute-phase glycoprotein, in one of the strongest noncovalent interactions known to occur naturally ${ }^{52}$.

Three types of haptoglobin polymers exist (FIG. 2), generated by combined expression of two alleles: HP1 and HP2. The three polymer types are referred to as HP1-1, HP1-2 and HP2-2. The functional differences between the different haptoglobin types are unclear and controversial (BOX 1). Functional differences might also differ between the CNS and the periphery as a result of CNS specialization (BOX 2). Resolving the controversies around the functional differences is important because haptoglobin could have potential as a therapeutic agent, and functional differences have implications for the selection of haptoglobin types to be tested in clinical trials.

Haptoglobin is mainly synthesized by the liver and reticuloendothelial system and is not normally synthesized within the brain. Instead, haptoglobin diffuses into the CSF from the blood, meaning that higher polymeric forms of the protein are not present in the CSF in the healthy state ${ }^{53}$ and levels of haptoglobin in the brain are much lower than levels in the blood in the healthy state ${ }^{54}$. Within each millilitre of intracranial blood, the molar amount of cell-free haemoglobin produced by haemolysis is approximately 250 -fold greater than the amount that the haptoglobin present in the same volume is able to bind to.

Current evidence points to two main functions of the haptoglobin-haemoglobin complex: protection against redox activity ${ }^{55-58}$ and haemoglobin clearance via the CD163 membrane receptor ${ }^{59}$. Crystal structure analyses of the haptoglobin-haemoglobin complex have revealed reactive iron and pro-oxidative tyrosine residues close to the haptoglobin-haemoglobin interface ${ }^{60}$, structural features that explain the ability of haptoglobin to prevent oxidative reactions and delay the release of haem. The binding of haptoglobin by haemoglobin exposes a neoepitope on the haptoglobin $\beta$-chain ${ }^{61}$ that enables CD163 to recognize the complex and initiate endocytosis. The affinity of CD163 for the haptoglobin-haemoglobin complex is tenfold higher than for uncomplexed haemoglobin ${ }^{62}$.

Some evidence suggests that astrocytes ${ }^{63}$ and oligodendrocytes ${ }^{64}$ express haptoglobin in pathological states. Given that oligodendrocytes and astrocytes are so abundant, local production of haptoglobin by these cells is an endogenous mechanism with potential to protect the brain against extravascular haemoglobin toxicity. However, this mechanism is clearly not enough, as most haemoglobin in the CSF after SAH is not bound to haptoglobin ${ }^{54}$. Furthermore, given that haptoglobin protects haemoglobin from auto-oxidation and that haemoglobin auto-oxidation interferes with haptoglobin binding, the scarcity of haptoglobin might render haemoglobin unscavengeable by CD163 and haptoglobin with time ${ }^{65}$; the extent to which this occurs requires further study.

One study has suggested that the lack of haptoglobin in the brain is compounded by saturation of the CD163 uptake system, as haptoglobin-haemoglobin complexes were detected in the CSF after SAH ${ }^{54}$. Intrathecal shedding of membrane-bound CD163 was also observed (FIC. 1), which resulted in high levels of soluble CD163 (sCD163) in the CSF after $\mathrm{SAH}^{54}$ and is likely to exacerbate saturation of the uptake system. However, sCD163 might be important in haptoglobin-independent pathways that detoxify free haemoglobin under conditions of severe haemolysis, such as haemorrhage. sCD163 and immunoglobulin $\mathrm{G}$ ( $\mathrm{IgG}$ ) can interact with free haemoglobin, and the sCD163-haemoglobin-IgG complex undergoes endocytosis into monocytes via the crystallizable fragment $(\mathrm{Fc})-\gamma$ receptor ${ }^{66}$.

\section{Haemopexin and CD91}

Unscavenged haemoglobin releases haem, which is sequestered with very high affinity by haemopexin ${ }^{67}$. Haemopexin is expressed by neurons and glia ${ }^{68}$; one study has shown that $\sim 90 \%$ of all haemopexin in the 


\section{Box 1 | Functional differences between haptoglobin types}

Many studies have investigated the functional differences between the HP1-1, HP1-2 and HP2-2 types of haptoglobin, which are expressed by homozygotes for the HP1 allele and heterozygotes and homozygotes for the HP2 allele, respectively; these studies have been reviewed elsewhere ${ }^{103}$. Expression levels differ reproducibly by type: haptoglobin expression is greater with the genotype HP1-1 than expression with the genotype HP1-2, which is in turn greater than that with the genotype HP2-2 (REF. ${ }^{203}$ ). Some studies ${ }^{204-206}$ have shown that HP1-1 is associated with better reduction of the redox potential of haemoglobin than is HP2-2 (REFS ${ }^{204-206}$ ), but other studies have not replicated this observation ${ }^{175,207,208}$. In complex with haemoglobin, HP2-2 seems to have a higher binding affinity for CD163 than does HP1-1 (REFS ${ }^{59,209}$ ). With respect to CD163-mediated cellular uptake of haptoglobin-haemoglobin complexes, different studies have indicated that HP1-1 is more effective, that HP2-2 is more effective and that they are equally effective ${ }^{175,209,210}$, suggesting that there is little or no difference in this aspect of haptoglobin function. Secretion of anti-inflammatory IL-10 in response to CD163 binding of the haptoglobin-haemoglobin complex differs according to type: secretion is several-fold higher with HP1-1 than with HP2-2 (REF. ${ }^{211}$ ).

Fenton reaction

A reaction in which $\mathrm{Fe}^{2+}$ causes disproportionation of a peroxide bond (in hydrogen peroxide or organic peroxides) to produce highly reactive hydroxyl $(\cdot \mathrm{OH})$ and

hydroperoxyl $(\cdot \mathrm{OOH})$ radicals.

Microparticles

Micrometre-sized membranebound vesicles released by a

variety of cell types;

microparticles retain the

molecules associated with, or embedded in, the original

membranes, and these molecules retain their function, thus acting in a paracrine fashion brain is produced intrathecally in the healthy state ${ }^{69}$ This production in the brain is in sharp contrast to that of haptoglobin, suggesting that the brain is geared towards clearing haem more effectively than haemoglobin. However, CSF levels of haemopexin are tenfold lower than levels in the circulation, suggesting that the capacity for haem binding in the brain is relatively low $^{69}$. The haem-haemopexin complex binds to CD91 (also known as LRP-1), which is expressed in neurons and glia ${ }^{70}$ and is subsequently cleared by endocytosis ${ }^{71}$ (FIG. 1). In a study of 30 patients with SAH, free haem was still detectable in the CSF after $\mathrm{SAH}^{69}$, indicating that the haemopexin-CD91 system cannot cope with the demands imposed on it after SAH.

After endocytosis by any cell type, haem is degraded by haem oxygenase, resulting in production of equimolar amounts of iron, carbon monoxide and biliverdin (FIG. 1). Biliverdin is then converted to bilirubin by biliverdin reductase $\mathrm{e}^{72}$. Two active isoforms of haem oxygenase exist: haem oxygenase 1, the expression of which is induced by haem and other forms of oxidative stress, mostly within glia, macrophages and endothelial cells ${ }^{73}$, and haem oxygenase 2 , which is constitutively expressed in most cell types, including neurons ${ }^{74}$. These enzymes are catalytically active only inside cells, as they require the microsomal NADPH-cytochrome P450 reductase to cleave haem ${ }^{72}$. Excess iron produced during this reaction is either transported out of cells (for example, via ferroportin ${ }^{75}$ ) or rapidly stored within ferritin - a heteropolymeric cage that can store up to 4,500 ferric ions as ferrihydrite aggregates in its core ${ }^{76}$ - in a safe but readily available form ${ }^{77}$. The reactive nature of noncomplexed iron means it has the potential to cause intracellular toxicity while it is shuttled inside cells. When the size of this so-called cellular labile iron pool ${ }^{78}$ increases, it can, for instance, participate in the Fenton reaction ${ }^{79}$ and activate the NLRP3 inflammasome ${ }^{80}$. Under conditions of iron overload, breakdown of ferritin within the lysosomes results in intracellular deposition of haemosiderin, which is insoluble but might not be redox inert ${ }^{76}$, so could be detrimental.

Haemoglobin uptake by macrophages converts them to a so-called Mhem phenotype, with antioxidative properties and reduced inflammatory cytokine expression $^{81,82}$ but still an undesirable overall profile that results in increased vascular permeability and microvascular inflammation ${ }^{83}$. Moreover, phagocytosis of iron-laden cell carcasses and debris results in iron-laden macrophages and microglia, which are pro-inflammatory ${ }^{84,85}$. Hence, although haemoglobin scavenging reduces the inflammatory effects of haemoglobin, some of these effects still occur.

\section{Haemoglobin in neurological disorders}

Extravascular haemoglobin and its breakdown products have been implicated in the pathophysiology of several neurological conditions, with evidence ranging from very strong in some to very weak in others. In the following sections, we review the evidence implicating haemoglobin in several conditions, focusing on conditions that produce macroscopic extravascular blood, in which the evidence is most robust. Animal studies are cited where evidence in humans is lacking or where experimental intervention has proven causality suggested by human observational data.

\section{Subarachnoid haemorrhage}

Neurological injury after SAH occurs in two stages: early brain injury (within $72 \mathrm{~h}$ ) and delayed brain injury, including delayed cerebral ischaemia, which presents days to weeks after haemorrhage. Early brain injury is associated with a transient increase in intracranial pressure and a decrease in cerebral blood flow, resulting in initiation of a toxic cascade that includes global cerebral ischaemia and cerebral oedema ${ }^{44,86,87}$. The presence of haemoglobin and its breakdown products in the brain along with the toxic cascade initiated during early brain injury is thought to have a key role in the development of delayed brain injury ${ }^{88}$.

Reported CSF concentrations of haemoglobin after SAH vary from $3 \mu \mathrm{M}$ to $250 \mu \mathrm{M}$ (REFS ${ }^{36,89-93}$ ). Some of this variation is likely to be due to the time points at which haemoglobin levels were measured and the specific species of haemoglobin being measured; the average of these reported values is $\sim 20 \mu \mathrm{M}$. Haem can also be detected in CSF after SAH; in one study, the concentration was $\sim 100 \mu \mathrm{g} / \mathrm{ml}\left(\mathrm{REF}^{69}{ }^{6}\right.$ ). Ferritin levels in the CSF also increase markedly as early as 1 day after SAH: the upper limit of the reference range is $12 \mathrm{ng} / \mathrm{ml}$, and the mean concentration on day 11 after SAH was $1,750 \mathrm{ng} / \mathrm{ml}$ $\left(\right.$ REFS $\left.^{94,95}\right)$. In addition, CSF iron levels increase from a mean of $2.3 \mu \mathrm{g} / \mathrm{dl}$ in controls to $27.9 \mu \mathrm{g} / \mathrm{dl}$ on day 5 after $\mathrm{SAH}$, with no apparent further increase ${ }^{95}$.

Haptoglobin levels in human CSF rise rapidly after $\mathrm{SAH}$, as expected after an injection of blood into the subarachnoid space, before declining, presumably owing to clearance of haptoglobin-haemoglobin complexes $^{96,97}$. A subsequent rise in haptoglobin levels has been observed, accompanied by a parallel rise in haemoglobin, suggesting saturation of the CD163-mediated scavenging pathway ${ }^{54,96}$; the precise contribution of intrathecal haptoglobin synthesis to the haptoglobin rise remains to be determined. Haptoglobin has been detected in association with microparticles in the CSF after SAH, and levels are higher in patients with vasospasm than in patients without ${ }^{97}$. The precise source 


\section{Box 2 | CNS specialization and haemoglobin scavenging}

The anatomical specialization of the brain reduces the first-line defence against the toxicity of extracellular haemoglobin via haptoglobin and CD163 scavenging for several reasons, discussed below.

\section{The blood-brain barrier}

The blood-brain barrier, although not an absolute barrier ${ }^{212}$, imposes a size restriction on molecules that can diffuse from the blood into the brain parenchyma ${ }^{213}$. Haptoglobin is mainly produced by the liver and granulocytes ${ }^{214}$ and is not normally synthesized within the intrathecal compartment. For these reasons, the presence of polymeric forms of haptoglobin (FIG. 2) depends on blood-brain barrier permeability ${ }^{53}$.

\section{Solute drainage}

The brain's drainage system for interstitial fluid and solutes is size-selective, and clearance of molecules with a molecular mass $>200 \mathrm{kDa}$ is limited ${ }^{215-217}$. The size of the simplest dimeric form of haptoglobin in complex with haemoglobin is below this threshold $(162 \mathrm{kDa})$, but the highest-order polymer of haptoglobin ${ }^{218}$ saturated with haemoglobin is $1,760 \mathrm{kDa}$. Drainage of ovalbumin-immunoglobulin $\mathrm{G}$ immune complexes with molecular masses of $500-2,000 \mathrm{kDa}\left(\mathrm{REF}^{219}\right)$ is severely impeded ${ }^{220}$; therefore, exit of haemoglobin in complex with larger haptoglobin species from the brain would be restricted.

\section{Immunological privilege}

In the uninflamed state, the brain contains few CD163-positive macrophages and no granulocytes ${ }^{221}$. During inflammation, myelomonocytic entry into the brain occurs but is delayed and low in magnitude ${ }^{222}$. These limitations could limit the number of CD163 binding sites available to scavenge haptoglobin-haemoglobin complexes ${ }^{54}$ and limit haptoglobin upregulation in the brain compared with elsewhere.

\section{Cellular traffic}

The reticuloendothelial system is responsible for haemoglobin clearance. Although a route for cellular traffic out of the brain via lymphatic drainage of the cerebrospinal fluid exists ${ }^{223}$, no high-throughput monocyte or macrophage exit system from the brain has been found. haemoglobin for CD163 in humans but not in mice ${ }^{59,62}$, so the mechanism underlying the observed difference in vasospasm between the transgenic and wild-type mice is unclear. In humans, haptoglobin facilitates haemoglobin uptake into cells, so differences in iron retention might occur if one haptoglobin isoform is better than the other at scavenging haemoglobin. Investigation of the CD91haem-haemopexin scavenging pathway after SAH in humans has produced surprising results. In one small prospective cohort study with 30 participants, CSF levels of haemopexin were elevated to a mean of $133.8 \mu \mathrm{g} / \mathrm{ml}$ in one-third of patients with SAH; the upper limit among healthy controls was $32.6 \mu \mathrm{g} / \mathrm{ml}$. Among the patients with elevated haemopexin levels, the rate of delayed cerebral ischaemia was higher and functional outcomes were poorer than among patients with normal haemopexin levels ${ }^{69}$. Given that haemopexin is presumed to have a neuroprotective effect, these results are unexpected, and further understanding of this system and its role in SAH is required.

A role for haem oxygenase 1 after SAH has been indicated by various studies. In one study, haem oxygenase 1 mRNA expression was increased in cells in human CSF, and this increase correlated with haematoma volume ${ }^{106}$. In small studies, elevated CSF levels of haem oxygenase after SAH in humans have been associated with an increased incidence of vasospasm ${ }^{92}$ and unfavourable functional outcomes ${ }^{93}$; however, as haem oxygenase 1 is an intracellular enzyme, these studies are complicated by the fact that detection of the enzyme might purely reflect tissue damage. By contrast, a similarly sized study of CSF from humans after SAH indicated a reduced incidence of vasospasm among patients with elevated levels of ferritin and bilirubin, which are products of haem oxygenase 1 activity ${ }^{95}$. In animal models of SAH, haem oxygenase 1 overexpression in brain tissue protected against vasospasm ${ }^{107}$ and suppression of haem oxygenase activity increased cerebral vasospasm and neuronal apoptosis ${ }^{106,108}$. The conflicting results in humans clearly demonstrate that the effects of haem oxygenase 1 activity in humans after SAH need further study. cohort study that included 133 patients, the HP2 allele was associated with increased cerebral salt wasting after SAH (OR 4.94, 95\% CI 1.78-17.43, $P=0.01)^{101}$. In one study that included 193 patients with SAH, the HP2 allele was also linked to worse functional outcomes ${ }^{102}$, although other smaller studies have not demonstrated this link ${ }^{99-101}$. A meta-analysis published in 2017 indicated that the HP2 allele confers a small effect on shortterm outcomes but not on long-term outcomes ${ }^{103}$, although the studies included were small, varied in design and did not include correction for prognostically relevant covariates. For these reasons, the conclusion should be viewed with caution until the results of an ongoing analysis of data from these studies at the individual patient level are known ${ }^{104}$, or until a larger, well-designed, prospective cohort study is conducted. In a mouse model of SAH, animals that were homozygous for a murine equivalent of the human HP2 allele experienced more marked cerebral vasospasm than wild-type mice that were homozygous for $H P 1^{105}$. However, the effect of haptoglobin differs between humans and mice: haptoglobin increases the affinity of

\section{Intracerebral haemorrhage} ondary neurological injury. The primary injury results from a rapid rise in brain volume and intracranial pressure due to haematoma formation. Secondary injury develops as a result of the physical effects of the initial haemorrhage, an inflammatory response to the haematoma and the release of blood breakdown products ${ }^{109}$. Perihaematomal oedema is a key component of the secondary injury; this phenomenon follows a triphasic pattern that comprises early ionic oedema followed by early vasogenic oedema and delayed vasogenic oedema. Delayed vasogenic oedema is thought to be driven by blood breakdown products ${ }^{43}$. Evidence from studies in humans and animals indicates that haemoglobin scavrhage and are linked to outcomes.

The haptoglobin-CD163 scavenging system has been shown to be active after intracerebral haemorrhage. In a prospective cohort study that had a low risk of bias
Intracerebral haemorrhage leads to primary and secenging pathways are active after intracerebral haemor- 
(Supplementary Table 1) and included 94 patients with intracerebral haemorrhage, the HP2 allele was associated with a reduced chance of a favourable outcome (OR $0.13, P=0.018$ ) after intracerebral haemorrhage but was not associated with mortality after intracerebral haemorrhage ${ }^{110}$. Evidence from animal models demonstrates that haptoglobin is protective after intracerebral haemorrhage, as induction of hypohaptoglobinaemia and genetic knockout of haptoglobin in rodent models results in worse neurological deficits after intracerebral haemorrhage than in untreated and wild-type animals ${ }^{64,111}$.

In human brain tissue harvested from around intracerebral haemorrhage haematomas ${ }^{112}$ and in animal models of intracerebral haemorrhage ${ }^{113}$, increased levels of CD163-positive microglia and macrophages have been detected. In a study of 54 patients with intracerebral haemorrhage, serum sCD163 levels above the average level in the group were associated with increased haematoma absorption and improved neurological recovery ${ }^{114}$. In an animal study, knockout of CD163 in a mouse model of intracerebral haemorrhage led to early beneficial effects but harmful late effects, indicating biphasic functions of CD163 in the mouse that are yet to be fully eludicated ${ }^{115}$. In murine neuronal cultures exposed to haemoglobin and after intracerebral injection of autologous blood in piglets, neuronal expression of CD163 was associated with neuronal toxicity, which was proposed to be related to neuronal uptake of toxic haemoglobin ${ }^{16,117}$. Neuronal CD163 expression has yet to be confirmed in humans. Nevertheless, the evidence together indicates that the haptoglobin-CD163 pathway has an important protective role in intracerebral haemorrhage. However, if manipulation of this pathway is considered as a therapeutic strategy, care must be taken to ensure that the potentially protective role of CD163positive microglia and macrophages is not counteracted by toxicity caused by iron overload in the brain.

Evidence from animal studies indicates that the haemopexin-CD91 scavenging pathway provides neuroprotection after intracerebral haemorrhage. In a study of a mouse model of intracerebral haemorrhage, haemopexin and CD91 levels within brain tissue were elevated, and administration of recombinant CD91 reduced various pathological indices and neurological deficits $^{118}$. In haemoglobin injection and collagenase injection mouse models of intracerebral haemorrhage, haemopexin knockout led to greater neuronal damage and worse outcomes than in mice that expressed haemopexin $^{119,120}$. Further supporting evidence comes from a study in which intracerebroventricular injection of a haemopexin-encoding recombinant adeno-associated viral vector to induce overexpression of haemopexin in a mouse model of intracerebral haemorrhage resulted in improved functional outcomes relative to outcomes in mice that received a control vector ${ }^{121}$.

The role of haem oxygenases in haemoglobin scavenging after intracerebral haemorrhage seems to vary according to the isoform. Upregulation of the inducible haem oxygenase 1 in the brain, notably in the microglia and astrocytes, has been demonstrated in animal models of intracerebral haemorrhage ${ }^{122-129}$. In a mouse model, selective overexpression of haem oxygenase 1 in astrocytes reduced mortality and improved short-term neurological outcomes up to 7 days after injury ${ }^{130,131}$. Pharmacological induction of haem oxygenase 1 with cobalt protoporphyrin IX in a mouse model of intracerebral haemorrhage was associated with worse neurological function in the short term (days 1-3) but improved neurological function at 28 days ${ }^{132}$ when compared with vehicle-treated mice. Conversely, haem oxygenase 1 knockout in a mouse model of intracerebral haemorrhage improved neurological function at $24 \mathrm{~h}$ after the insult in comparison with outcomes in wild-type mice, although this benefit was no longer apparent at $72 \mathrm{~h}$ $\left(\right.$ REF $\left.^{73}\right)$. This evidence suggests that haem oxygenase 1 has a protective effect after intracerebral haemorrhage, but the enzyme might have harmful effects in the short term.

Investigation of the effects of haem oxygenase 2 in intracerebral haemorrhage has been performed in mouse knockout models, but the findings are conflicting. In the collagenase injection model of intracerebral haemorrhage, haem oxygenase 2 knockout has been associated with increased neuronal damage ${ }^{133,134}$. By contrast, in the blood injection model, haem oxygenase 2 knockout was protective, with a weak and variable effect on neurological outcome ${ }^{135,136}$. Studies in stroma-free haemoglobin injection models of intracerebral haemorrhage have yielded similarly conflicting results: haem oxygenase 2 knockout has been associated with increased ${ }^{119}$ and decreased ${ }^{136}$ neuronal damage. The metabolism of haem over time might provide an explanation for the different outcomes in such models.

\section{Traumatic brain injury}

Traumatic brain injury (TBI) can be complicated by the presence of extravascular blood within one or more of the extradural, subdural, subarachnoid, intraventricular or intraparenchymal compartments. Blood breakdown products are thought to lead to secondary brain injury ${ }^{137}$; therefore, haem and haemoglobin scavenging might have a key role in minimizing this injury after TBI. The presence of intracranial blood is also a risk factor for the development of post-traumatic seizures ${ }^{138}$, and the free haemoglobin and reactive oxygen species that it produces are thought to play an important role ${ }^{2,139}$. Furthermore, as for aneurysmal SAH, traumatic SAH can lead to cerebral vasospasm and delayed neurological deficits, although the clinical manifestation of vasospasm after traumatic SAH is less severe and the pathogenesis might be different ${ }^{140}$.

The role of haptoglobin after TBI is not well defined. In humans, haptoglobin levels are elevated in the serum $^{141}$ and the $\mathrm{CSF}^{142}$ after TBI, and the main source of elevated haptoglobin in TBI is thought to be the liver ${ }^{141}$. Homozygous expression of the HP1 allele has been associated with worse neuropsychological outcomes (assessed with the Verbal Intelligence Quotient and Finger Tapping Test) at 1 month and 12 months after TBI in humans relative to outcomes in people with other HP genotypes, but the association was not observed at 6 months or 10 years after injury ${ }^{143}$. In an adult mouse model of TBI, haptoglobin-knockout mice exhibited fewer neurological deficits than did wild-type mice, but lesion volumes did not differ ${ }^{144}$. In the same model in 
older adult mice, haptoglobin-knockout mice had larger lesion volumes but neurological deficits did not differ from those of wild-type mice ${ }^{144}$. This evidence indicates that age-dependent effects of haptoglobin might influence outcomes after $\mathrm{TBI}^{144}$. Immunoreactive CD163positive cells are elevated in perilesional areas in a rat TBI model, suggesting that haemoglobin scavenging mechanisms are upregulated after $\mathrm{TBI}^{145}$.

Whether haptoglobin influences post-traumatic seizures is also unclear. One study has been conducted to investigate the association of haptoglobin genotype with post-traumatic seizures ${ }^{143}$. This study included only 50 patients but demonstrated no significant association between haptoglobin genotype and the development of post-traumatic seizures after TBI. Similarly, little is known about the role of haptoglobin in vasospasm after traumatic SAH. Determining whether haptoglobin does affect these outcomes is an area of interest for future research.

Animal studies suggest that haem oxygenases are involved in the response to TBI. Haem oxygenase 1 levels are elevated after TBI in animal models ${ }^{146-148}$. In an adult mouse model, knockout of haem oxygenase 2 was associated with significantly greater neuronal loss after TBI than in wild-type mice ${ }^{149}$. Haem oxygenase 2 knockout in an immature rat model was associated with larger lesions at 1 week after injury, but not at 2 weeks, than in wild-type mice, and a tendency towards worse neurological recovery ${ }^{150}$.

\section{Superficial siderosis}

Superficial siderosis is a neurodegenerative condition characterized by chronic low-grade subarachnoid bleeding from a wide range of sources, whether microscopic or macroscopic ${ }^{151}$. A human pathological study has revealed an intense reaction of microglia, astrocytes and Bergmann glia, and the presence of axonal debris ${ }^{3}$. Experimental studies in rabbits have shown that the glial reaction is related to the RBC fraction of blood, indicating that haemoglobin is the likely culprit ${ }^{152}$. In addition, pronounced upregulation of haem oxygenase 1 and ferritin as well as iron deposition are seen in superficial siderosis in humans ${ }^{3}$ and animals ${ }^{152,153}$. Intracisternal administration of the haem oxygenase inhibitor tin protoporphyrin in rabbits decreased iron accumulation but not the microglial response ${ }^{153}$. Whether HP genotype and the efficiency of haemoglobin scavenging affect clinical outcomes in superficial siderosis is unclear but is the subject of an ongoing study ${ }^{154}$.

\section{Other conditions}

The conditions discussed above are associated with macroscopic release of blood into the CNS. A more gradual build-up of extracorpuscular haemoglobin has been explored as part of the aetiology of several other neurological conditions. For instance, raised serum levels of free haemoglobin polypeptides have been demonstrated in patients with multiple sclerosis and have been shown to correlate with neurodegeneration ${ }^{5}$. Elevated haemoglobin levels have been identified in brain tissue from humans with Alzheimer disease ${ }^{4}$, and these levels correlated with amyloid burden ${ }^{155}$. This observation raises the possibility that circulating free haemoglobin enters the CNS through a leaky BBB to modify disease course. Some evidence has also linked free haemoglobin to idiopathic generalized epilepsy and Parkinson disease, but the evidence (Supplementary Box 1) is either sparse or conflicting in all these conditions, and further research is required.

\section{Haemoglobin as a therapeutic target}

Several treatment strategies are intended to clear haemoglobin or mitigate its toxic and other deleterious consequences, and many of these strategies have been studied (Supplementary Table 2). In this section, we discuss these strategies, focusing on their application in SAH and intracerebral haemorrhage, the conditions in which most evidence has shown haemoglobin to mediate toxicity.

\section{Physical augmentation of clearance}

An extravasated blood clot acts as a static source of large amounts of extracellular haemoglobin, so its surgical removal is a rational therapeutic approach to prevent the harmful consequences of cell-free haemoglobin. Reduction of the clot burden in SAH via direct surgical removal has shown promise in a primate model of $\mathrm{SAH}$, in which removal was associated with reduced vasospasm ${ }^{156}$. However, in this model, the clot was focally placed in the Sylvian fissure; reproducing complete evacuation in real SAH, in which blood is distributed more widely, is challenging owing to the anatomy of the subarachnoid space. Indeed, the rate of clot resolution in a study of 413 patients with SAH does not seem to differ between conventional surgery and coiling ${ }^{157}$.

Intrathecal thrombolytics have also been used to clear the blood clot. A meta-analysis of the five available randomized controlled trials (RCTs) showed that administration of thrombolytics (tissue plasminogen activator or urokinase) rather than a placebo or no treatment was associated with a significant reduction in angiographic vasospasm, delayed cerebral ischaemia, poor outcome and chronic hydrocephalus ${ }^{158}$. However, the analysed data were from generally small exploratory studies in which limited blinding measures were used; therefore, the risk of bias was considerable. Furthermore, in one of the studies, an intrathecal vasodilator was administered with the thrombolytic, which might have accounted for some of the improvement in outcome ${ }^{159}$. In an RCT that included 60 patients with $\mathrm{SAH}$, intraventricular thrombolysis with concomitant low-frequency head-motion therapy after SAH did not lead to clinical improvements ${ }^{160}$. However, in a small RCT that included 12 patients with SAH, intrathecal thrombolysis (compared with placebo) worsened inflammation, an effect that could attenuate the benefits of rapid clot clearance ${ }^{161}$. A new trial that will include 440 patients has been registered and is designed to conclusively address the efficacy of intrathecal thrombolysis ${ }^{162}$. Recruitment of patients with SAH to a phase I trial of an alternative approach to clot removal has begun in the United States; insertion of a closed-loop lumbar intrathecal filtration device will be used to remove $\mathrm{RBCs}$ in the $\mathrm{CSF}^{163}$. 
An alternative approach to physical clearance of haemoglobin is CSF diversion via a ventriculostomy or lumbar drain to increase CSF drainage and reduce CSF levels of haemoglobin. Retrospective studies showed that use of a lumbar drain after SAH lowered the incidence of delayed cerebral ischaemia and improved outcomes ${ }^{164,165}$. In another study of patients with SAH, the incidence of vasospasm and patient outcomes did not differ whether lumbar drainage or ventricular drainage was used ${ }^{166}$. Only one prospective study of CSF diversion via lumbar drainage in nonhydrocephalic patients with SAH has been conducted ${ }^{167}$. This high-quality RCT, which was at low risk of bias (Supplementary Table 2), included 210 patients and showed that lumbar drainage (versus no drainage) reduced the incidence of delayed cerebral ischaemia from $35 \%$ to $21 \%$ but had no effect on long-term outcomes ${ }^{168}$.

The above findings suggest that the effects of CSF diversion are limited, at least in nonhydrocephalic patients, as the diverted CSF would have otherwise drained through endogenous routes. Furthermore, the use of CSF drainage devices will not have an appreciable effect on the local tissue concentrations of haemoglobin and its metabolites adjacent to the solid clot, as the clot continues to release haemoglobin. Nevertheless, studies conducted to date might have lacked the power necessary to detect a difference in clinical outcome, and larger studies might reveal a long-term benefit of CSF drainage. In one large prospective RCT that is underway, known as EARLYDRAIN, the effects of lumbar drainage within $72 \mathrm{~h}$ of the ictus will be studied; recruitment of 300 patients is complete, and the results are forthcoming ${ }^{168}$.

Mechanical evacuation of the clot in intracerebral haemorrhage is equally as controversial as in SAH. Clot volume can be similar to that after SAH, but intracerebral haemorrhage affects a much smaller surface area than SAH; in the former, the clot is focal within the brain parenchyma, whereas in the latter, the blood clot coats the surface of the cortex. Two large, randomized clinical trials that included a total of 1,634 patients with intracerebral haemorrhage showed no benefit of early surgical evacuation compared with initial medical treatment ${ }^{169,170}$. Although the primary outcome was clear, its generalizability is more complex. Patients who were assigned to initially receive medical treatment were allowed to cross over to surgery in the following hours or days if their clinical condition changed, which might have hampered attempts to demonstrate benefits of surgical clot evacuation because these patients remained in the initial conservative treatment group in the primary intention-to-treat analysis. Furthermore, at the time these trials were designed, most neurosurgeons were of the opinion that at least some patients with intracerebral haemorrhage would benefit from clot removal. As a result, randomization for all patients was felt to be ethically unacceptable, so the studies only included patients for whom the benefit of clot removal was considered by neurosurgeons to be uncertain; if a neurosurgeon felt confident that a patient would benefit from clot evacuation, they were not entered into the trial. There is no practical way to resolve whether these excluded patients would benefit from surgery or not. As a result, despite a lack of objective evidence, many clinicians continue to feel that a subgroup of patients with superficial, moderate-sized clots might benefit from clot reduction. However, whether any benefit of mechanical clot removal arises from a reduction of intracranial pressure or a reduction in toxicity mediated by blood products is unclear. A phase II trial of thrombolysis with recombinant tissue-type plasminogen activator administered through a catheter into the haematoma cavity showed that the reduction in blood volume reduces oedema ${ }^{171}$. A phase III trial for which recruitment of 500 patients has been completed will hopefully clarify whether a reduction in clot burden improves outcomes ${ }^{172}$.

\section{Augmentation of haemoglobin scavenging}

Haptoglobin. Given that endogenous haptoglobin is consumed and depleted after haemorrhage or haemolysis, haptoglobin supplementation is an appealing treatment strategy ${ }^{54}$. A preliminary study of intrathecal administration (topical at the time of surgery or postoperatively via a cisternal drain) of haptoglobin as a treatment for vasospasm was conducted in 27 patients with SAH in 1979 in Japan, and improvements in vasospasm in many patients suggested some therapeutic benefits ${ }^{173}$. However, the study design is best described as an open-label, uncontrolled study, which precludes any strong conclusions being drawn. A personal communication from a member of the medical team at that time suggests that the treatment was abandoned after one patient developed seizures (M. Miyaoka, personal communication). Furthermore, the haptoglobin used in this study was derived from pooled blood donations, and given the low proportion of individuals who are homozygous for the HP1 allele (which evidence suggests is even lower in Japan, where the blood was sourced ${ }^{174}$ ), the patients in the study probably received large amounts of HP2-2. The haptoglobin phenotype of patients was not determined; therefore, any patients included who expressed HP1-1 might not have benefited or might even have been harmed as a result of immunological rejection. Thus, the role of haptoglobin administration for vasospasm prophylaxis and improvement of outcome is not known.

One haptoglobin product, which is $\sim 90 \%$ HP2-2 (REF. ${ }^{175}$ ), is approved in Japan for clinical use as a therapeutic plasma protein for intravenous infusion in patients with haemoglobinaemia associated with intravascular haemolysis in response to a thermal burn, blood transfusion and cardiopulmonary bypass. In a study published in 2017, intraoperative administration of haptoglobin was associated with a lower risk of acute kidney injury in patients undergoing cardiovascular surgery ${ }^{176}$. However, there are currently no neurological indications for treatment with haptoglobin.

The possibility of haptoglobin supplementation as a treatment for patients with SAH is an avenue for further research, but many issues remain to be resolved. More certainty is needed about which haptoglobin type HP1-1 or HP2-2 - is most beneficial. The mechanism by which endogenous haptoglobin improves outcome is unknown. No clinical haptoglobin preparation is yet 
available, and the optimal concentration and mode of delivery to the site of the clot need to be worked out. No point-of-care test to determine haptoglobin phenotype is available, and the immunogenicity of allogeneic haptoglobin infusion, which might affect its efficacy, has not been systematically studied, although from a safety perspective, only 3 of 4,600 clinically overt adverse events related to blood product transfusion in Japan over an 8 -year period were associated with anti-haptoglobin antibodies ${ }^{177}$. The effects of increased protein load in the CNS as a result of haptoglobin infusion are unknown, and the risk of increased iron load due to improved uptake also needs to be determined.

Haemopexin. Unlike haptoglobin, haemopexin is constitutively expressed in the healthy brain, yet its levels in the CSF are still low compared with its levels in the blood. Preclinical data (see Intracerebral haemorrhage above) indicate that intrathecal administration of haemopexin or selective agonists of haemopexin expression are possible therapeutic strategies to neutralize haem toxicity after brain haemorrhage, although no obvious clinical candidates currently exist.

Transcriptional upregulation of antioxidative responses. Genes that encode proteins that are essential for haemoglobin clearance and redox protection are under the transcriptional control of signalling pathways that sense oxidative stress and inflammation (FIG. 3). One of the main transcriptional regulators of the host response to haemoglobin is nuclear factor erythroid 2-related factor 2 (NRF2; encoded by the NFE2L2 gene). In the brain, NRF2 upregulates haptoglobin expression in oligodendrocytes $^{64}$ and CD36-mediated phagocytosis of RBCs ${ }^{47}$. In animal models of SAH, administration of the NRF2 activator sulforaphane, compared with administration of vehicle alone, reduced cerebral vasospasm, brain oedema, BBB leakage, cortical apoptosis and motor deficits $^{178-180}$. This evidence makes NRF2 a candidate target for treatment of SAH. Multiple NRF2 activators have been studied in SAH; one of the most potent and widely studied is sulforaphane. Practically, sulforaphane has limited stability at room temperature, but when complexed with cyclodextrin, its stability is improved, which makes it suitable for clinical use, and a phase II clinical trial of this complex in 90 patients with SAH is currently underway ${ }^{181}$.

Another global transcriptional regulator with antiinflammatory and antioxidant properties ${ }^{182}$ is peroxisome proliferator-activated receptor- $\gamma$ (PPAR $\gamma$; encoded by the PPARG gene). PPAR $\gamma$ inactivates NF- $\mathrm{KB}$ and is a transcriptional activator of the genes that encode NRF2 and other redox-protective proteins, such as catalase ${ }^{182}$. In an animal model of intracerebral haemorrhage, PPAR $\gamma$ activators enhanced haematoma clearance by increasing microglial phagocytic activity and upregulating CD36 (REF. $\left.{ }^{183}\right)$. In a rat model of SAH, the PPAR $\gamma$ agonist rosiglitazone reduced vasospasm and improved outcomes $^{184}$. On the basis of this evidence, a phase II study (SHRINC) of the related drug pioglitazone has been started to assess its effect on the rate of haematoma resolution after intracerebral haemorrhage ${ }^{185}$.
Some evidence also suggests that co-activation of NRF2 and PPAR $\gamma$ might have additional therapeutic potential. PPARG contains an NRF2 response element ${ }^{186}$ and NFE2L 2 contains a PPAR $\gamma$ response element ${ }^{187}$, so expression of each can activate expression of the other ${ }^{182}$.

Mitigation of haemoglobin redox toxicity. The iron chelator deferiprone, which can cross the $\mathrm{BBB}$, has been studied as a potential therapeutic agent in disorders associated with haemoglobin toxicity. In one small dose escalation study that included 20 patients, an intravenous dose of deferiprone up to $62 \mathrm{mg} / \mathrm{kg}$ daily for 3 days after intracerebral haemorrhage seemed to be safe ${ }^{188}$. However, an RCT of deferiprone at the same intravenous dose for 5 days was halted owing to concerns over acute respiratory distress syndrome ${ }^{189}$. A multicentre phase III RCT of a lower dose $(32 \mathrm{mg} / \mathrm{kg}$ daily for 3 days) in 294 patients is now underway ${ }^{190}$. A previous study that included 42 patients has suggested that intravenous deferiprone at $32 \mathrm{mg} / \mathrm{kg}$ daily for 3 days reduces intracerebral haemorrhage oedema compared with no treatment ${ }^{191}$. This study was well designed and at relatively low risk of bias for its size (Supplementary Table 2) but was not sufficiently powered to examine clinical outcomes, which were similar in both arms at 30 days after intracerebral haemorrhage. The effects of deferiprone will also be studied in SAH in an RCT that has been registered ${ }^{192}$.

Deferiprone has also been studied in superficial siderosis. A small (10 patients), single-arm, open-label study of $30 \mathrm{mg} / \mathrm{kg}$ oral deferiprone daily for 90 days suggested a satisfactory safety profile and decreased haemosiderin deposition assessed with $\mathrm{MRI}^{193}$. This study was followed by a larger and longer observational study of 38 patients, 30 of whom completed the follow-up ${ }^{194}$. After 2 years of deferiprone treatment, 19 patients reported no disease progression or an improvement in at least one neurological domain; disease progression would be expected over this time period. In half of the 16 patients for whom a complete MRI assessment was available in this study, a reduction in haemosiderin was observed over the 2 years. Another single-arm, nonrandomized study of deferiprone in superficial siderosis is ongoing ${ }^{195}$. Although the observations to date are promising, the nature of such small open-label studies means they are at high risk of bias.

Several other drugs have potential for reducing haemoglobin redox toxicity and have either been investigated or are under active investigation (BOX 3). Perhaps the most robustly investigated therapeutic agent for $\mathrm{SAH}$ is tirilazad, a nonglucocorticoid 21-aminosteroid that inhibits lipid peroxidation. However, a Cochrane analysis of five trials of tirilazad, which were assessed as being at low risk of bias, had high follow-up rates and involved a total of 3,821 patients with $\mathrm{SAH}$, identified no clinical benefit ${ }^{196}$.

Modulation of inflammation. Inflammation after intracerebral haemorrhage or SAH can be modulated in multiple ways, and many therapeutic avenues are currently under investigation. Most are not specific to haemoglobin scavenging, but one potential treatment is IL-1 receptor antagonist (IL1-RA), which 
a In physiological conditions

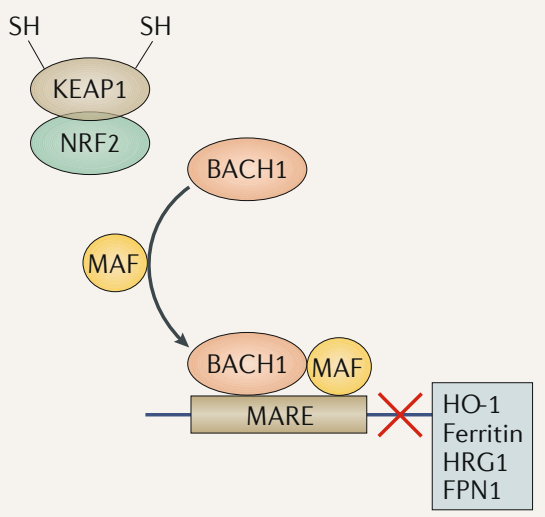

b Under oxidative stress

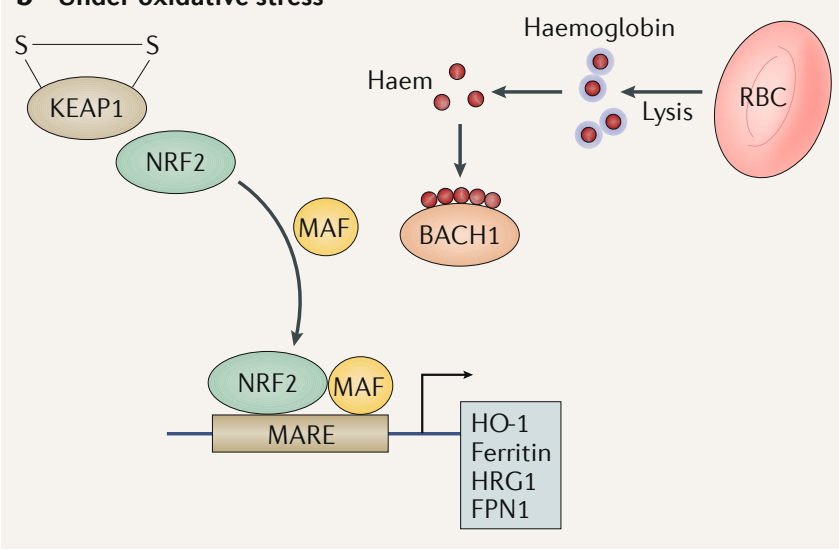

C Pro-inflammatory molecules

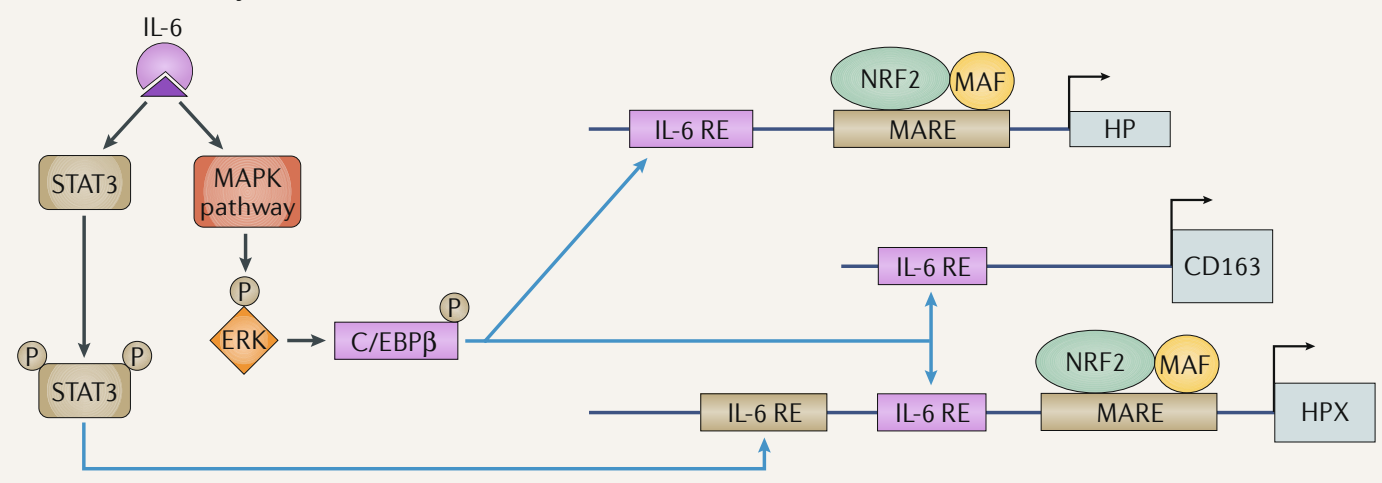

d Anti-inflammatory molecules

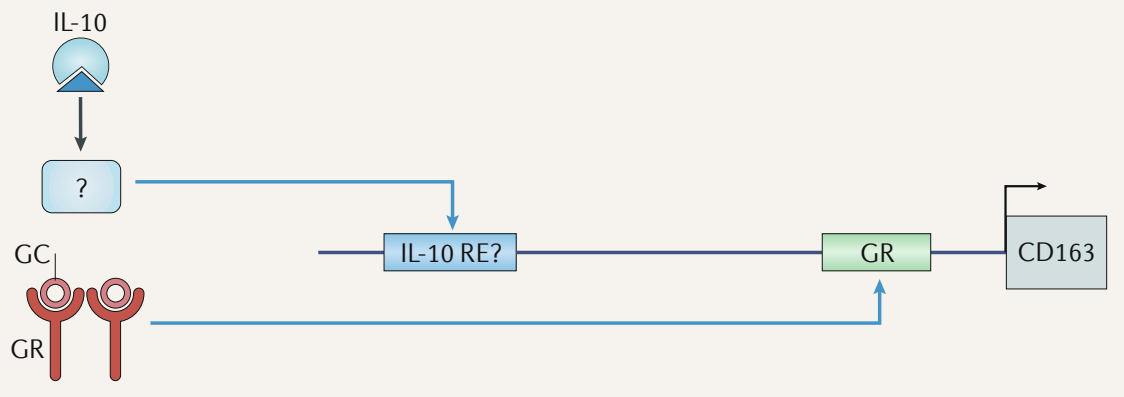

Fig. 3 | Transcriptional regulation of genes involved in haemoglobin scavenging and redox protection. One of the main transcriptional regulators of genes involved in haemoglobin scavenging and redox protection is nuclear factor erythroid 2-related factor 2 (NRF2), a redox-sensitive transcription factor that binds to response elements (REs) upstream of a wide range of such genes ${ }^{237}$. a In physiological conditions, NRF2 is targeted for ubiquitylation by Kelch-like $\mathrm{ECH}$-associated protein 1 (KEAP1), and transcriptional regulator protein $\mathrm{BACH} 1$ can bind to V-maf musculoaponeurotic fibrosarcoma oncogene homologue (MAF), which enables it to bind to MAF recognition elements (MAREs). BACH1 binds to a subset of NFR2 REs and suppresses transcription of the downstream genes. $\mathbf{b}$ | Under oxidative stress, KEAP1 is inactivated by oxidation of sulfhydryl (thiol) groups on certain cysteine residues; internal disulfide bridge formation is depicted as an example, but other reactions between KEAP1 and NRF2 inducers can occur, such as alkylation of the cysteine thiol groups. Upon KEAP1 inactivation, NRF2 is released. Red blood cell (RBC) lysis leads to haem binding of $\mathrm{BACH} 1$, causing $\mathrm{BACH} 1$ to dissociate from MAREs. NRF2 is therefore able to bind to MAF and MAREs, activating transcription of protective genes, such as those that encode haem oxygenase 1 (HO1), ferritin heavy and light chains, haem-responsive gene 1 protein homologue (HRG1; also known as SLC48A1) and ferroportin 1 (FPN1; also known as SLC40A1). c | During inflammation, release of IL-6 leads to phosphorylation of signal transducer and activator of transcription 3 (STAT3) and CCAAT/enhancer-binding protein- $\beta$ (C/EBP $\beta$ ), which bind to IL-6 REs that activate transcription of downstream genes involved in haemoglobin scavenging, including those that encode haptoglobin (HP), CD163 and haemopexin (HPX). NRF2 is upregulated in response to inflammation and cerebral insults ${ }^{238}$ and can bind to MAREs that activate transcription of the same genes. $\mathbf{d}$ | Anti-inflammatory molecules, such as IL-10 and glucocorticoids (GCs), upregulate transcription of the CD163 gene. Question marks indicate uncertain elements of the pathway. GR, glucocorticoid receptor; GRE, glucocorticoid response element; ERK, extracellular-signal-regulated kinase; MAPK, mitogen-activated protein kinase. 


\section{Box 3 | Drugs with potential for reducing haemoglobin redox toxicity}

\section{Minocycline}

Minocycline is an antibiotic but is also an iron chelator and antioxidant. Extensive safety data are available for this drug, and small clinical studies to determine its effects after intracerebral haemorrhage and subarachnoid haemorrhage (SAH) are ongoing ${ }^{224-226}$.

\section{Selenium and paracetamol}

Trials of selenium ${ }^{227}$ and paracetamol ${ }^{228}$ to reduce oxidative stress after $\mathrm{SAH}$ were registered $>10$ years ago, but the status of these trials is unclear, and no results have been published to date.

\section{Hydrogen-rich saline}

Exogenous hydrogen supplementation with intraperitoneal administration of hydrogenrich saline in a rat model of SAH reduced oxidative stress and inflammatory markers, whereas physiological saline did not ${ }^{229}$; on this basis, an early clinical trial of orally administered hydrogen-rich water versus regular water after $\mathrm{SAH}$ is underway ${ }^{230}$.

\section{NXY-059}

NXY-059 is a free radical scavenger that has been tested in a commercially sponsored randomized controlled trial involving 603 patients with intracerebral haemorrhage. The results were reported as a safety study and concluded that NXY-059 is safe ${ }^{231}$, but no reductions in deaths or poor outcomes were noted and no further progress has been made.

\section{Ferrostatin 1, liproxstatin and zileuton}

Ferrostatin 1 was identified in 2012 as a specific inhibitor of ferroptosis ${ }^{232}$ and inhibits neuronal death as a result of haemoglobin and free $\mathrm{Fe}^{2+}\left(\mathrm{REF}^{233}\right)$. In a mouse model of intracerebral haemorrhage, ferrostatin 1 treatment was associated with reduced lesion volumes and improvements in neurological function ${ }^{233}$. These findings have been replicated with the ferroptosis inhibitors liproxstatin 1 and zileuton ${ }^{234}$. Their effects are thought to result predominantly from free radical trapping but also lipoxygenase inhibition ${ }^{235}$. Owing to the relative novelty of these agents, no human studies have been conducted. Zileuton is a good candidate for a clinical study, as it is currently used as maintenance therapy for asthma.

\section{Necrostatin 1}

Necrostatin 1 (NEC-1) is a necroptosis inhibitor. After intraventricular administration in an animal model of intracerebral haemorrhage, NEC-1 reduced haematoma volume, cell death and oedema, and improved neurobehavioural outcomes ${ }^{236}$.

specifically reduces haem-induced neuronal death in organotypic slice cultures ${ }^{16}$. A dose-finding study in 25 patients with SAH demonstrated brain penetration with therapeutic levels of IL1-RA and good safety ${ }^{197}$. A further single-blinded, open-label study that included 136 patients with SAH has demonstrated that IL1-RA reduces inflammatory markers ${ }^{198}$, and the drug is currently being trialled in a definitive RCT aiming to recruit 1,000 patients with $\mathrm{SAH}^{199}$.

Increasing nitric oxide bioavailability. Nitric oxide donors could be beneficial after intracerebral haemorrhage and $\mathrm{SAH}$, as they could mitigate the adverse effects of nitric oxide scavenging by haemoglobin. Sodium nitroprusside and glyceryl trinitrate are the nitric oxide donors that have been studied most, but both have profound vasodilatory effects and induce hypotension, which is not tolerable after SAH because cerebral perfusion could be compromised. Sodium nitrite is currently the only clinical option; a study of this drug in 18 patients with SAH showed that it is safe and produced adequate therapeutic levels ${ }^{200}$, but a clinical trial that was subsequently started has been terminated for unknown reasons ${ }^{201}$. After intracerebral haemorrhage, more-aggressive blood pressure management is recommended; glyceryl trinitrate has been trialled in this context, albeit for the purpose of blood pressure management ${ }^{202}$. Regardless, this treatment did not improve patient outcomes.

\section{Future directions}

A large body of preclinical work has investigated different approaches to ameliorating the secondary brain injury caused by extracellular haemoglobin. Despite promising early results for several agents, few have undergone the further preclinical evaluation required before translation to human studies. A handful of agents have been tested in small clinical studies, but few of these studies have been sufficiently powered to conclusively assess clinical benefits to patients with intracerebral haemorrhage or SAH. Therefore, the need for further research in this area is pressing.

To address this need, the Haemoglobin After Intracranial Haemorrhage (HATCH) consortium held a meeting to arrive at a consensus opinion regarding the immediate priorities for research in intracranial haemolysis that will deliver clinical benefits. The HATCH consortium was formed by invitation of all groups who had published studies of haptoglobin in patients with intracranial haemorrhage and includes groups from the United States, Japan and Europe. Four main research priorities were identified, discussed below.

\section{Improved understanding of mechanisms}

The first priority identified was a need for improved basic scientific understanding of the mechanisms of haemoglobin toxicity and its clearance in the brain. Although the basic structures of many of the pathways involved have been described, little is known about the details of their control, their interrelationships and their functional relevance. Examples of aspects that need to be studied are genetic control of expression of haemoglobin scavengers, the kinetics of haemoglobin and its scavengers within the brain, and the relationship between haemoglobin scavenging and inflammation. Specific clinical-grade therapeutics, such as haptoglobin, are needed for preclinical and phase I and phase II human studies. Research might reveal that multiple levels in the haemoglobin and haem scavenging pathways need to be targeted. In order to study these basic mechanisms and evaluate new therapeutics, current preclinical models need improvement. These models need to recapitulate the clearance pathways that are relevant in humans and exhibit consistent functional outcomes that equate to clinical scenarios. Such models will need to be crossvalidated between laboratories.

\section{Prospective studies}

Research into basic mechanisms will need to be complemented by large, multicentre, prospective observational cohort studies of intracranial haemorrhage that examine the impact of functionally distinct genetic variants of elements of the haemoglobin clearance pathways on clinical outcomes while controlling for covariates that affect prognosis and for ancestral background. Large studies of genetic predisposition to aneurysm formation and the occurrence of 
intracerebral haemorrhage have been conducted, but only small, underpowered genetic association studies have been used to assess the clinical outcomes of SAH and intracerebral haemorrhage.

\section{Disease-specific outcome measures}

The success of prospective studies relies on the development of robust disease-specific outcome measures. Outcomes after SAH and intracerebral haemorrhage can be thought of in simple terms as being influenced by a combination of primary brain injury and secondary brain injury mediated through extracellular haemoglobin. Current scales for assessing brain injury, such as the modified Rankin scale and the Glasgow Outcome Scale, might be well suited to capture the initial catastrophic injury that results from shearing and a surge in intracranial pressure after very large bleeds, but sensitive measures of long-term cognitive and psychosocial functioning are needed. Such outcome measures are also essential for the success of any future clinical trials of therapeutics.

\section{Point-of-care genotyping}

The success of clinical trials might also depend on appropriate risk stratification. Effective stratification might require the development of rapid point-of-care tests for genetic determinants of outcome, such as haptoglobin genotype. These tests might also be of wider clinical benefit in prognostication after intracranial haemorrhage.

\section{Conclusions}

Following intracranial haemorrhage, RBCs lyse and release their contents, initiating a cascade of events that results in secondary injury. Extracellular haemoglobin has been identified as a major early mediator of these events. Multiple endogenous mechanisms can mitigate haemoglobin-related pathology in humans; these mechanisms include erythrophagocytosis, the haptoglobin-CD163 pathway, the haemopexin-CD91 pathway and the intracellular haem oxygenases. These systems differ between humans and animals, and between the CNS and the periphery, and detailed functional implications of these differences require further study. The role of extracellular haemoglobin in secondary brain injury means that it is an attractive therapeutic target to minimize such injury and improve outcomes. Several therapeutic approaches are feasible, and many are under investigation, but robust clinical studies are currently lacking. Therefore, further research is needed, and the HATCH consortium has identified four areas of priority for research: improved understanding of toxic and clearance mechanisms, prospective observational studies in intracranial haemorrhage, the development of disease-specific outcome measures, and the development of point-of-care tests for risk stratification. Advances in these areas would facilitate the translation of knowledge of haemoglobin toxicity and clearance mechanisms into clinical benefits.

Published online 20 June 2018
1. Regan, R. F. \& Panter, S. S. Neurotoxicity of hemoglobin in cortical cell culture. Neurosci. Lett. 153, 219-222 (1993).

2. Rosen, A. D. \& Frumin, N. V. Focal epileptogenesis after intracortical hemoglobin injection. Exp. Neurol. 66, 277-284 (1979).

3. Koeppen, A. H. et al. The pathology of superficial siderosis of the central nervous system. Acta Neuropathol. 116, 371-382 (2008). This article presents the most detailed pathological study of haemoglobin toxicity accompanying superficial siderosis.

4. Slemmon, J. R., Hughes, C. M., Campbell, G. A. \& Flood, D. G. Increased levels of hemoglobin-derived and other peptides in Alzheimer's disease cerebellum. J. Neurosci. 14, 2225-2235 (1994).

5. Lewin, A et al. Free serum haemoglobin is associated with brain atrophy in secondary progressive multiple sclerosis. Wellcome Open Res. 1 , 10 (2016)

This is an intriguing study showing that extracellular haemoglobin might be important in neurological conditions that are not characterized by macroscopic bleeding.

6. Reeder, B. J., Svistunenko, D. A., Cooper, C. E. \& Wilson, $\mathrm{M}$. T. The radical and redox chemistry of myoglobin and hemoglobin: from in vitro studies to human pathology. Antioxid. Redox Signal. 6 , 954-966 (2004)

7. Marnett, L. J., Riggins, J. N. \& West, J. D. Endogenous generation of reactive oxidants and electrophiles and their reactions with DNA and protein. J. Clin. Invest. 111, 583-593 (2003).

8. Reeder, B. J. et al. Toxicity of myoglobin and haemoglobin: oxidative stress in patients with rhabdomyolysis and subarachnoid haemorrhage. Biochem Soc. Trans. 30, 745-748 (2002).

9. Suzuki, N. et al. Identification of 5-hydroxy eicosatetraenoic acid in cerebrospinal fluid after subarachnoid hemorrhage. J. Neurochem. 41 1186-1189 (1983)

10. Balla, G., Jacob, H. S., Eaton, J. W., Belcher, J. D. \& Vercellotti, G. M. Hemin: a possible physiological mediator of low density lipoprotein oxidation and endothelial injury. Arterioscler Thromb. 11 1700-1711 (1991)
11. Yang W. S. \& Stockwell, B. R. Ferroptosis: death by lipid peroxidation. Trends Cell Biol. 26, 165-176 (2016).

12. Kwon, M. S. et al. Methemoglobin is an endogenous toll-like receptor 4 ligand-relevance to subarachnoid hemorrhage. Int. J. Mol. Sci. 16, 5028-5046 (2015).

13. Figueiredo, R. T. et al. Characterization of heme as activator of Toll-like receptor 4. J. Biol. Chem. 282, 20221-20229 (2007).

14. Grootjans, S., Vanden Berghe, T. \& Vandenabeele, P. Initiation and execution mechanisms of necroptosis: an overview. Cell Death Differ. 24, 1184-1195 (2017).

15. Dutra, F. F. et al. Hemolysis-induced lethality involves inflammasome activation by heme. Proc. Natl Acad. Sci. USA 1 11, E4110-E4118 (2014).

16. Greenhalgh, A. D. et al. Interleukin-1 receptor antagonist is beneficial after subarachnoid haemorrhage in rat by blocking haem-driven inflammatory pathology. Dis. Model. Mech. 5, 823-833 (2012).

This study shows that haem causes inflammasome activation after $\mathrm{SAH}$

17. Joshi, M. S. et al. Nitric oxide is consumed, rather than conserved, by reaction with oxyhemoglobin under physiological conditions. Proc. Natl Acad. Sci. USA 99, 10341-10346 (2002).

18. Kajita, Y. et al. Combined effect of L-arginine and superoxide dismutase on the spastic basilar artery after subarachnoid hemorrhage in dogs. J. Neurosurg. 80, 476-483 (1994).

19. Zou, M. H., Shi, C. \& Cohen, R. A. Oxidation of the zinc-thiolate complex and uncoupling of endothelial nitric oxide synthase by peroxynitrite. J. Clin. Invest 109, 817-826 (2002).

20. Sabri, M. et al. Uncoupling of endothelial nitric oxide synthase after experimental subarachnoid hemorrhage. J. Cereb. Blood Flow Metab. 31, 190-199 (2011)

21. Faraci, F. M. \& Brian, J. E. Jr. Nitric oxide and the cerebral circulation. Stroke 25,692-703 (1994).

22. Voetsch, B., Jin, R. C. \& Loscalzo, J. Nitric oxide insufficiency and atherothrombosis. Histochem. Cell Biol. 122, 353-367 (2004).
23. Suzuki, S. et al. Cerebral microthrombosis in symptomatic cerebral vasospasm - a quantitative histological study in autopsy cases. Neurol. Med. Chir 30, 309-316 (1990).

24. Friedrich, B., Muller, F., Feiler, S., Scholler, K. \& Plesnila, N. Experimental subarachnoid hemorrhage causes early and long-lasting microarterial constriction and microthrombosis: an in-vivo microscopy study. J. Cereb. Blood Flow Metab. 32, 447-455 (2012)

25. Stein S. C., Browne K. D. Chen, X. H Smith D. H. $\mathrm{X}$ Graham, D. I. Thromboembolism and delayed cerebral ischemia after subarachnoid hemorrhage: an autopsy study. Neurosurgery 59, 781-787; discussion 787-788 (2006).

26. Petzold, G. C. et al. Nitric oxide modulates spreading depolarization threshold in the human and rodent cortex. Stroke 39, 1292-1299 (2008).

27. Dreier, J. P. et al. Nitric oxide scavenging by hemoglobin or nitric oxide synthase inhibition by $\mathrm{N}$-nitro-L-arginine induces cortical spreading ischemia when $\mathrm{K}^{+}$is increased in the subarachnoid space. J. Cereb. Blood Flow Metab. 18, 978-990 (1998).

28. Sehba, F. A., Schwartz, A. Y., Chereshnev, I. \& Bederson, J. B. Acute decrease in cerebral nitric oxide evels after subarachnoid hemorrhage. J Cereb. Blood Flow Metab. 20, 604-611 (2000)

29. Kanamaru, K., Waga, S., Kojima, T., Fujimoto, K. \& Niwa, S. Endothelium-dependent relaxation of canine basilar arteries. Part 2: inhibition by hemoglobin and cerebrospinal fluid from patients with aneurysmal subarachnoid hemorrhage. Stroke 18, 938-943 (1987)

30. Byrne, J. V., Griffith, T. M., Edwards, D. H., Harrison, T. J. $\&$ Johnston, K. R. Investigation of the vasoconstrictor action of subarachnoid haemoglobin in the pig cerebral circulation in vivo. Br. J. Pharmacol. 97 669-674 (1989)

31. Iqbal, S. et al. Inducible nitric oxide synthase (NOS-2) in subarachnoid hemorrhage: regulatory mechanisms and therapeutic implications. Brain Circ. 2, 8-19 (2016).

32. Woszczyk, A., Deinsberger, W. \& Boker, D. K. Nitric oxide metabolites in cisternal CSF correlate with cerebral vasospasm in patients with a subarachnoid 
haemorrhage. Acta Neurochir.145, 257-263; discussion 263-254(2003).

33. Suzuki, Y. et al. Nitric oxide metabolites in the cisternal cerebral spinal fluid of patients with subarachnoid hemorrhage. Neurosurgery 41 807-811; discussion 811-802 (1997).

34. Suzuki, M. et al. Increased levels of nitrite/nitrate in the cerebrospinal fluid of patients with subarachnoid hemorrhage. Neurosurg. Rev. 22, 96-98 (1999).

35. Sadamitsu, D. et al. Cerebrospinal fluid and plasma concentrations of nitric oxide metabolites in postoperative patients with subarachnoid hemorrhage. Crit. Care Med. 29, 77-79 (2001).

36. Rejdak, K. et al. Cerebrospinal fluid nitrite/nitrate correlated with oxyhemoglobin and outcome in patients with subarachnoid hemorrhage. J. Neurol. Sci. 219, 71-76 (2004).

37. Ng, W. H., Moochhala, S., Yeo, T. T., Ong, P. L. \& $\mathrm{Ng}$, P. Y. Nitric oxide and subarachnoid hemorrhage elevated level in cerebrospinal fluid and their implications. Neurosurgery 49, 622-626; discussion 626-627 (2001)

38. Ding, H. Y., Han, X., Lv, C. Z. \& Dong, O. Xenon-CT study of regional cerebral blood flow around hematoma in patients with basal ganglia hemorrhage. Acta Neurochir. Suppl. 105, 161-164 (2008).

39. Kalita, J., Misra, U. K., Ranjan, P., Pradhan, P. K. \& Das, B. K. Effect of mannitol on regional cerebral blood flow in patients with intracerebral hemorrhage. J. Neurol. Sci. 224, 19-22 (2004).

40. Huang, F. P. et al. Brain edema after experimental intracerebral hemorrhage: role of hemoglobin degradation products. J. Neurosurg. 96, 287-293 (2002).

41. Katsu, M. et al. Hemoglobin-induced oxidative stress contributes to matrix metalloproteinase activation and blood-brain barrier dysfunction in vivo. J. Cereb. Blood Flow Metab. 30, 1939-1950 (2010).

42. Rosenberg, G. A. \& Navratil, M. Metalloproteinase inhibition blocks edema in intracerebral hemorrhage in the rat. Neurology 48, 921-926 (1997).

43. Urday, S. et al. Targeting secondary injury in intracerebral haemorrhage-perihaematomal oedema. Nat. Rev. Neurol. 11, 111-122 (2015)

44. Claassen, J. et al. Global cerebral edema after subarachnoid hemorrhage: frequency, predictors, and impact on outcome. Stroke 33, 1225-1232 (2002).

45. Johshita, H., Kassell, N. F., Sasaki, T. \& Ogawa, H. Impaired capillary perfusion and brain edema following experimental subarachnoid hemorrhage: a morphometric study. J. Neurosurg. 73, 410-417 (1990).

46. Fadok, V. A., Warner, M. L., Bratton, D. L. \& Henson, P. M. CD36 is required for phagocytosis of apoptotic cells by human macrophages that use either a phosphatidylserine receptor or the vitronectin receptor (alpha v beta 3). J. Immunol. 161, 6250-6257 (1998)

47. Zhao, X. et al. Cleaning up after ICH: the role of Nrf2 in modulating microglia function and hematoma clearance. J. Neurochem. 133, 144-152 (2015).

48. Knutson, M. D., Oukka, M., Koss, L. M., Aydemir, F. \& Wessling-Resnick, M. Iron release from macrophages after erythrophagocytosis is up-regulated by ferroportin 1 overexpression and down-regulated by hepcidin. Proc. Natl Acad. Sci. USA 102, 1324-1328 (2005).

49. Yang, Z. et al. Kinetics and specificity of feline leukemia virus subgroup $C$ receptor (FLVCR) export function and its dependence on hemopexin. J. Biol. Chem. 285 28874-28882 (2010).

50. Kondo, H., Saito, K., Grasso, J. P. \& Aisen, P. Iron metabolism in the erythrophagocytosing Kupffer cell. Hepatology 8, 32-38 (1988)

51. Bhakdi, S. \& Tranum-Jensen, J. On the cause and nature of C9-related heterogeneity of terminal complement complexes generated on target erythrocytes through the action of whole serum. J. Immunol. 133, 1453-1463 (1984)

52. Nagel, R. L. \& Gibson, Q. H. The binding of hemoglobin to haptoglobin and its relation to subunit dissociation of hemoglobin. J. Biol. Chem. 246, 69-73 (1971).

53. Chamoun, V. et al. Haptoglobins as markers of blood-CSF barrier dysfunction: the findings in normal CSF. J. Neurol. Sci. 182, 117-121 (2001).

54. Galea, J. et al. The intrathecal CD163-haptoglobinhemoglobin scavenging system in subarachnoid hemorrhage. J. Neurochem. 121, 785-792 (2012). The first detailed description of the CD163haptoglobin-haemoglobin scavenging system in the human brain, in healthy individuals and after SAH.
55. Cooper, C. E. et al. Haptoglobin binding stabilizes hemoglobin ferryl iron and the globin radical on tyrosine beta145. Antioxid. Redox Signal. 18, 2264-2273 (2013).

56. Buehler, P. W. et al. Haptoglobin preserves the CD163 hemoglobin scavenger pathway by shielding hemoglobin from peroxidative modification. Blood 113, 2578-2586 (2009).

57. Boretti, F. S. et al. Sequestration of extracellular hemoglobin within a haptoglobin complex decreases its hypertensive and oxidative effects in dogs and guinea pigs. J. Clin. Invest. 119, 2271-2280 (2009).

58. Banerjee, S. et al. Haptoglobin alters oxygenation and oxidation of hemoglobin and decreases propagation of peroxide-induced oxidative reactions. Free Radic. Biol. Med. 53, 1317-1326 (2012).

59. Kristiansen, M. et al. Identification of the haemoglobin scavenger receptor. Nature 409, 198-201 (2001). A crystal-clear description of how CD163, the receptor for haemoglobin-haptoglobin complexes, was discovered.

60. Andersen, C. B. et al. Structure of the haptoglobinhaemoglobin complex. Nature 489, 456-459 (2012)

61. Nielsen, M. J. et al. A unique loop extension in the serine protease domain of haptoglobin is essential for CD163 recognition of the haptoglobin-hemoglobin complex. J. Biol. Chem. 282, 1072-1079 (2007).

62. Etzerodt, A. et al. Plasma clearance of hemoglobin and haptoglobin in mice and effect of CD 163 gene targeting disruption. Antioxid. Redox Signal. 18, 2254-2263 (2013).

63. Lee, M. Y. et al. Upregulation of haptoglobin in reactive astrocytes after transient forebrain ischemia in rats. J. Cereb. Blood Flow Metab. 22, 1176-1180 (2002).

64. Zhao, X. et al. Neuroprotective role of haptoglobin after intracerebral hemorrhage. J. Neurosci. 29.

15819-15827 (2009).

This is the first experimental study to demonstrate the therapeutic value of haptoglobin and NRF2 activation in intracerebral haemorrhage.

65. Vallelian, F. et al. The reaction of hydrogen peroxide with hemoglobin induces extensive alpha-globin crosslinking and impairs the interaction of hemoglobin with endogenous scavenger pathways. Free Radic. Biol. Med. 45, 1150-1158 (2008).

66. Subramanian, K., Du, R., Tan, N. S., Ho, B. \& Ding, J. L. CD163 and IgC codefend against cytotoxic hemoglobin via autocrine and paracrine mechanisms. J. Immunol. 190, 5267-5278 (2013).

67. Paoli, M. et al. Crystal structure of hemopexin reveals a novel high-affinity heme site formed between two beta-propeller domains. Nat. Struct. Biol. 6, 926-93 (1999).

68. Morris, C. M., Candy, J. M., Edwardson, J. A Bloxham, C. A. \& Smith, A. Evidence for the localization of haemopexin immunoreactivity in neurones in the human brain. Neurosci. Lett. 149 , 141-144 (1993)

69. Garland, P. et al. Heme-hemopexin scavenging is active in the brain and associates with outcome after subarachnoid hemorrhage. Stroke 47, 872-876 (2016).

70. Moestrup, S. K., Gliemann, J. \& Pallesen, G. Distribution of the alpha 2-macroglobulin receptor/low density lipoprotein receptor-related protein in human tissues. Cell Tissue Res. 269, 375-382 (1992).

71. Hvidberg, V. et al. Identification of the receptor scavenging hemopexin-heme complexes. Blood 106, 2572-2579 (2005).

72. Maines, M. D. The heme oxygenase system: a regulator of second messenger gases. Annu. Rev. Pharmacol Toxicol 37, 517-554 (1997).

73. Wang, J. \& Doré, S. Heme oxygenase- 1 exacerbates early brain injury after intracerebral haemorrhage. Brain 130, 1643-1652 (2007).

74. Ewing, J. F. \& Maines, M. D. In situ hybridization and immunohistochemical localization of heme oxygenase- 2 mRNA and protein in normal rat brain: differential distribution of isozyme 1 and 2. Mol. Cell Neurosci. 3, 559-570 (1992)

75. Boserup, M. W., Lichota, J., Haile, D. \& Moos, T. Heterogenous distribution of ferroportin-containing neurons in mouse brain. Biometals 24, 357-375 (2011).

76. Harrison, P. M. \& Arosio, P. The ferritins: molecular properties, iron storage function and cellular regulation. Biochim. Biophys. Acta 1275, 161-203 (1996).

77. Kidane, T. Z., Sauble, E. $\&$ Linder, M. C. Release of iron from ferritin requires lysosomal activity. $\mathrm{Am}$. J. Physiol. Cell Physiol. 291, C445-C455 (2006).
78. Breuer, W., Shvartsman, M. \& Cabantchik, Z. I. Intracellular labile iron. Int. J. Biochem. Cell Biol. 40 350-354 (2008).

79. Spangler, B. et al. A reactivity-based probe of the intracellular labile ferrous iron pool. Nat. Chem. Biol. 12, 680-685 (2016).

80. Nakamura, K. et al. Activation of the NLRP3 inflammasome by cellular labile iron. Exp. Hematol. 44, 116-124 (2016)

81. Boyle, J. J. et al. Activating transcription factor 1 directs Mhem atheroprotective macrophages through coordinated iron handling and foam cell protection. Circ. Res. 110, 20-33 (2012)

82. Chinetti-Gbaguidi, G., Colin, S. \& Staels, B. Macrophage subsets in atherosclerosis. Nat. Rev. Cardiol. 12, 10-17 (2015).

83. Guo, L. et al. CD163+ macrophages promote angiogenesis and vascular permeability accompanied by inflammation in atherosclerosis. J. Clin. Invest. 128, 1106-1124 (2018)

84. Sindrilaru, A. et al. An unrestrained proinflammatory M1 macrophage population induced by iron impairs wound healing in humans and mice. J. Clin. Invest. 121, 985-997 (2011).

85. Zhang, X., Surguladze, N., Slagle-Webb, B., Cozzi, A. \& Connor, J. R. Cellular iron status influences the functional relationship between microglia and oligodendrocytes. Glia 54, 795-804 (2006).

86. Schubert, G. A., Seiz, M., Hegewald, A. A., Manville, J. $\&$ Thome, C. Hypoperfusion in the acute phase of subarachnoid hemorrhage. Acta Neurochir. Suppl. 110, 35-38 (2011)

87. Bederson, J. B., Germano, I. M. \& Guarino, L. Cortical blood flow and cerebral perfusion pressure in a new noncraniotomy model of subarachnoid hemorrhage in the rat. Stroke 26, 1086-1091; discussion 1091-1082 (1995).

88. Brathwaite, S. \& Macdonald, R. L. Current management of delayed cerebral ischemia: update from results of recent clinical trials. Trans/ Stroke Res. 5, 207-226 (2014).

89. Dunne, V. G. \& Besser, M. Outcome scoring in neurosurgery: the cognitive outcome score, an alternative to the Glasgow outcome scale. J. Clin. Neurosci. 12, 119-123 (2005).

90. Dunne, V. G., Bhattachayya, S., Besser, M., Rae, C. \& Griffin, J. L. Metabolites from cerebrospinal fluid in aneurysmal subarachnoid haemorrhage correlate with vasospasm and clinical outcome: a patternrecognition 1 H NMR study. NMR Biomed. 18, 24-33 (2005).

91. Mast, H., Poche, H. \& Marx, P. Hemoglobin quantitation in subarachnoid hemorrhage. Klin Wochenschr. 65, 513-515 (1987).

92. Pyne-Geithman, G. J. et al. Bilirubin production and oxidation in CSF of patients with cerebral vasospasm after subarachnoid hemorrhage. J. Cereb. Blood Flow Metab. 25, 1070-1077 (2005).

93. Wang, K. C. et al. Prognostic value of intrathecal heme oxygenase- 1 concentration in patients with fisher grade III aneurysmal subarachnoid hemorrhage. J. Neurosurg. 121, 1388-1393 (2014).

94. Petzold, A. et al. Cerebrospinal fluid ferritin level, a sensitive diagnostic test in late-presenting subarachnoid hemorrhage. J. Stroke Cerebrovasc. Dis. 20, 489-493 (2011).

95. Suzuki, H., Muramatsu, M., Kojima, T. \& Taki, W. Intracranial heme metabolism and cerebral vasospasm after aneurysmal subarachnoid hemorrhage. Stroke 34, 2796-2800 (2003).

96. Durnford, A. et al. Haemoglobin scavenging after subarachnoid haemorrhage. Acta Neurochir. Suppl. 120, 51-54 (2015)

97. Przybycien-Szymanska, M. M., Yang, Y. \& Ashley, W. W. Microparticle derived proteins as potential biomarkers for cerebral vasospasm post subarachnoid hemorrhage. A preliminary study. Clin. Neurol. Neurosurg. 141, 48-55 (2016).

98. Borsody, M., Burke, A., Coplin, W., Miller-Lotan, R. \& Levy, A. Haptoglobin and the development of cerebral artery vasospasm after subarachnoid hemorrhage. Neurology 66, 634-640 (2006).

This article presents the first clinical study to show an association between haptoglobin genotype and outcome after SAH.

99. Leclerc, J. L. et al. Haptoglobin phenotype predicts the development of focal and global cerebral vasospasm and may influence outcomes after aneurysmal subarachnoid hemorrhage. Proc. Natl Acad. Sci. USA 112, 1155-1160 (2015).

100. Ohnishi, H. et al. Haptoglobin phenotype predicts cerebral vasospasm and clinical deterioration after 
aneurysmal subarachnoid hemorrhage. J. Stroke Cerebrovasc. Dis. 22, 520-526 (2013)

101. Murthy, S. B. et al. Haptoglobin 2-2 genotype is associated with cerebral salt wasting syndrome in aneurysmal subarachnoid hemorrhage. Neurosurgery 78, 71-76 (2016).

102. Kantor, E. et al. Haptoglobin genotype and functional outcome after aneurysmal subarachnoid hemorrhage. J. Neurosurgery. 120, 386-390 (2014)

103. Gaastra, B., Glazier, J., Bulters, D. \& Galea, I. Haptoglobin genotype and outcome after subarachnoid haemorrhage: new insights from a metaanalysis. Oxid. Med. Cell Longev. 2017, 6747940 (2017).

A meta-analysis of all published clinical studies linking haptoglobin genotype and outcome after SAH.

104. Gaastra, B. et al. Haptoglobin after subarachnoid haemorrhage: individual patient level data (IPLD) analysis. NHS http://www.crd.york.ac.uk/PROSPERO display record.php?ID=CRD 42017070830 (2017).

105. Chaichana, K. L., Levy, A. P., Miller-Lotan, R., Shakur, S. \& Tamargo, R. J. Haptoglobin 2-2 genotype determines chronic vasospasm after experimental subarachnoid hemorrhage. Stroke 38, 3266-3271 (2007).

106. Schallner, N. et al. Microglia regulate blood clearance in subarachnoid hemorrhage by heme oxygenase- 1 . J. Clin. Invest. 125, 2609-2625 (2015).

107. Ono, S., Komuro, T. \& Macdonald, R. L. Heme oxygenase-1 gene therapy for prevention of vasospasm in rats. J. Neurosurg. 96, 1094-1102 (2002)

108. Suzuki, H. et al. Heme oxygenase-1 gene induction as an intrinsic regulation against delayed cerebral vasospasm in rats. J. Clin. Invest. 104, 59-66 (1999).

109. Keep, R. F., Hua, Y. \& Xi, G. Intracerebral haemorrhage: mechanisms of injury and therapeutic targets. Lancet Neurol. 11, 720-731 (2012).

110. Murthy, S. B. et al. Presence of haptoglobin-2 allele is associated with worse functional outcomes after spontaneous intracerebral hemorrhage. World Neurosurg. 83, 583-587 (2015)

111. Zhao, X. et al. Cytoprotective role of haptoglobin in brain after experimental intracerebral hemorrhage. Acta Neurochir Suppl. 111, 107-112 (2011).

112. Liu, B. et al. CD163/Hemoglobin oxygenase- 1 pathway regulates inflammation in hematoma surrounding tissues after intracerebral hemorrhage. J. Stroke Cerebrovasc. Dis. 24 , 2800-2809 (2015)

113. Cao, S. et al. Hematoma changes during clot resolution after experimental intracerebral hemorrhage. Stroke 47, 1626-1631 (2016).

114. Xie, W. J., Yu, H. Q., Zhang, Y., Liu, Q. \& Meng, H. M. CD163 promotes hematoma absorption and improves neurological functions in patients with intracerebral hemorrhage. Neural Regen Res. 11 1122-1127 (2016)

115. Leclerc, J. L. et al. The absence of the CD163 receptor has distinct temporal influences on intracerebral hemorrhage outcomes. J Cereb. Blood Flow Metab. 38, 262-273 (2017)

116. Liu, R. et al. CD163 expression in neurons after experimental intracerebral hemorrhage. Stroke $\mathbf{4 8}$ 1369-1375 (2017)

117. Chen-Roetling, J. \& Regan, R. F. Haptoglobin increases the vulnerability of CD163-expressin neurons to hemoglobin. J. Neurochem. 139, 586-595 (2016)

118. Wang, G. et al. Low-density lipoprotein receptorrelated protein-1 facilitates heme scavenging after intracerebral hemorrhage in mice. $J$. Cereb. Blood Flow Metab. 37, 1299-1310 (2017).

119. Ma, B. et al. Deletion of the hemopexin or heme oxygenase-2 gene aggravates brain injury following stroma-free hemoglobin-induced intracerebral hemorrhage J. Neuroinflamm. 13, 26 (2016).

120. Chen, L., Zhang, X., Chen-Roetling, J. \& Regan, R. F. Increased striatal injury and behavioral deficits after intracerebral hemorrhage in hemopexin knockout mice. J. Neurosurg. 114, 1159-1167 (2011)

121. Leclerc, J. L. et al. Increased brain hemopexin levels improve outcomes after intracerebral hemorrhage. J. Cereb. Blood Flow Metab. https://doi.org/10.1177/0 271678X16679170 (2016)

This is the first experimental study to demonstrate the therapeutic value of haemopexin after intracerebral haemorrhage.

122. Shang, H. et al. Time course of Keap1-Nrf2 pathway expression after experimental intracerebral haemorrhage: correlation with brain oedema and neurological deficit. Free Radic. Res. 47, 368-375 (2013).

123. Wang, G. et al. Time course of heme oxygenase- 1 and oxidative stress after experimental intracerebral hemorrhage. Acta Neurochir. 153, 319-325 (2011).

124. Chen, M. \& Regan, R. F. Time course of increased heme oxygenase activity and expression after experimental intracerebral hemorrhage: correlation with oxidative injury. J. Neurochem. 103, 2015-2021 (2007).

125. Wu, J. et al. Iron and iron-handling proteins in the brain after intracerebral hemorrhage. Stroke 34, 2964-2969 (2003).

126. Wagner, K. R. et al. Protein oxidation and heme oxygenase-1 induction in porcine white matter following intracerebral infusions of whole blood or plasma. Dev. Neurosci. 24, 154-160 (2002)

127. Wu, J. et al. Oxidative brain injury from extravasated erythrocytes after intracerebral hemorrhage. Brain Res. 953, 45-52 (2002).

128. Nakaso, K. et al. Co-induction of heme oxygenase- 1 and peroxiredoxin I in astrocytes and microglia around hemorrhagic region in the rat brain. Neurosci. Lett. 293, 49-52 (2000).

129. Matz, P. G., Weinstein, P. R. \& Sharp, F. R. Heme oxygenase- 1 and heat shock protein 70 induction in glia and neurons throughout rat brain after experimental intracerebral hemorrhage. Neurosurgery 40, 152-160; discussion 160-152 (1997).

130. Chen-Roetling, J., Song, W., Schipper, H. M., Regan, C. S. $\&$ Regan, R. F. Astrocyte overexpression of heme oxygenase-1 improves outcome after intracerebral hemorrhage. Stroke 46, 1093-1098 (2015).

131. Chen-Roetling, J. et al. Astrocyte heme oxygenase-1 reduces mortality and improves outcome after collagenase-induced intracerebral hemorrhage. Neurobiol. Dis. 102, 140-146 (2017).

132. Zhang, Z. et al. Distinct role of heme oxygenase- 1 in early- and late-stage intracerebral hemorrhage in 12-month-old mice. J. Cereb. Blood Flow Metab. 37, 25-38 (2017)

133. Wang, J., Zhuang, H. \& Doré, S. Heme oxygenase 2 is neuroprotective against intracerebral hemorrhage. Neurobiol. Dis. 22, 473-476 (2006).

134. Wang, J. \& Doré, S. Heme oxygenase 2 deficiency increases brain swelling and inflammation after intracerebral hemorrhage. Neuroscience $\mathbf{1 5 5}$, 1133-1141 (2008)

135. Chen-Roetling, J., Cai, Y. \& Regan, R. F. Neuroprotective effect of heme oxygenase-2 knockout in the blood injection model of intracerebral hemorrhage. BMC Res. Notes 7, 561 (2014)

136. Qu, Y., Chen, J., Benvenisti-Zarom, L., Ma, X. \& Regan, R. F. Effect of targeted deletion of the heme oxygenase-2 gene on hemoglobin toxicity in the striatum. J. Cereb. Blood Flow Metab. 25, 1466-1475 (2005).

137. Lok, J. et al. Intracranial hemorrhage: mechanisms of secondary brain injury. Acta Neurochir. Suppl. 111, 63-69 (2011)

138. Verellen, R. M. \& Cavazos, J. E. Post-traumatic epilepsy: an overview. Therapy 7, 527-531 (2010).

139. Meyerhoff, J. L., Lee, J. K., Rittase, B. W., Tsang, A. Y. \& Yourick, D. L. Lipoic acid pretreatment attenuates ferric chloride-induced seizures in the rat. Brain Res. 1016, 139-144 (2004).

140. Kramer, D. R., Winer, J. L., Pease, B. A., Amar, A. P. \& Mack, W. J. Cerebral vasospasm in traumatic brain injury. Neurol. Res. Int. 2013, 415813 (2013).

141. Yang, S. et al. Elevated serum haptoglobin after traumatic brain injury is synthesized mainly in liver. J. Neurosci. Res. 91, 230-239 (2013).

142. Conti, A. et al. Proteome study of human cerebrospinal fluid following traumatic brain injury indicates fibrin(ogen) degradation products as trauma-associated markers. J. Neurotrauma 21, 854-863 (2004).

143. Anderson, G. D. et al. Haptoglobin phenotype and apolipoprotein E polymorphism: relationship to posttraumatic seizures and neuropsychological functioning after traumatic brain injury. Epilepsy Behav 16, 501-506 (2009).

144. Glushakov, A. V., Arias, R. A., Tolosano, E. \& Doré, S. Age-dependent effects of haptoglobin deletion in neurobehavioral and anatomical outcomes following traumatic brain injury. Front. Mol. Biosci. 3, 34 (2016).

145. Zhang, Z., Zhang, Z. Y., Wu, Y. \& Schluesener, H. J. Lesional accumulation of CD163+ macrophages/ microglia in rat traumatic brain injury. Brain Res. 1461, 102-110 (2012).
146. Okubo, S., Xi, G., Keep, R. F., Muraszko, K. M. \& Hua, Y. Cerebral hemorrhage, brain edema, and heme oxygenase-1 expression after experimental traumatic brain injury. Acta Neurochir. Suppl. 118, 83-87 (2013).

147. Liu, Y., Zhang, Z., Luo, B. \& Schluesener, H. J. Lesional accumulation of heme oxygenase- $1+$ microglia/ macrophages in rat traumatic brain injury. Neuroreport 24, 281-286 (2013).

148. Fukuda, K. et al. Induction of heme oxygenase-1 (HO-1) in glia after traumatic brain injury. Brain Res. 736, 68-75 (1996)

149. Chang, E. F. et al. Heme oxygenase-2 protects against lipid peroxidation-mediated cell loss and impaired motor recovery after traumatic brain injury. J. Neurosci. 23, 3689-3696 (2003).

150. Yoneyama-Sarnecky, T. et al. Heme oxygenase- 2 modulates early pathogenesis after traumatic injury to the immature brain. Dev. Neurosci. 32, 81-90 (2010).

151. Levy, M., Turtzo, C. \& Llinas, R. H. Superficial siderosis: a case report and review of the literature. Nat. Clin. Pract. Neurol. 3, 54-58; quiz 59 (2007)

152. Koeppen, A. H. \& Borke, R. C. Experimental superficial siderosis of the central nervous system I. Morphological observations. J. Neuropathol. Exp. Neurol. 50, 579-594 (1991).

153. Koeppen, A. H. \& Dickson, A. C. Tin-protoporphyrin prevents experimental superficial siderosis in rabbits. J. Neuropathol. Exp. Neurol. 61, 689-701 (2002).

154. National Health Service Research Authority. Clearing haemoglobin in superficial siderosis (CHISS). NHS https://www.hra.nhs.uk/planning-and-improvingresearch/application-summaries/research-summaries/ clearing-haemoglobin-in-superficial-siderosis-chiss/ (2016).

155. Wu, C. W. et al. Hemoglobin promotes Abeta oligomer formation and localizes in neurons and amyloid deposits. Neurobiol. Dis. 17, 367-377 (2004).

156. Handa, Y. et al. The effect of timing of clot removal on chronic vasospasm in a primate model. J. Neurosurg. 67, 558-564 (1987).

157. Ibrahim, G. M. et al. Method of aneurysm treatment does not affect clot clearance after aneurysmal subarachnoid hemorrhage. Neurosurgery $\mathbf{7 0}$, 102-109; discussion 109 (2012).

158. Kramer, A. H. \& Fletcher, J. J. Locally-administered intrathecal thrombolytics following aneurysmal subarachnoid hemorrhage: a systematic review and meta-analysis. Neurocrit. Care. 14, 489-499 (2011).

159. Hänggi, D. et al. A multimodal concept in patients after severe aneurysmal subarachnoid hemorrhage: results of a controlled single centre prospective randomized multimodal phase I/II trial on cerebra vasospasm. Cent. Eur. Neurosurg. 70, 61-67 (2009).

160. Etminan, N. et al. Prospective, randomized, open-label phase II trial on concomitant intraventricular fibrinolysis and low-frequency rotation after severe subarachnoid hemorrhage. Stroke 44, 2162-2168 (2013).

161. Kramer, A. H. et al. Intraventricular fibrinolysis with tissue plasminogen activator is associated with transient cerebrospinal fluid inflammation: a randomized controlled trial. J. Cereb. Blood Flow Metab. 35, 1241-1248 (2015).

162. US National Library of Medicine. ClinicalTrials.gov https://www.clinicaltrials.gov/ct2/show/NCT03187405 (2017).

163. US National Library of Medicine. ClinicalTrials. govhttps://www.clinicaltrials.gov/ct2/show/ NCT02872636 (2018)

164. Klimo, P., Kestle J. R MacDonald, J. D \& Schmidt, R. H. Marked reduction of cerebra vasospasm with lumbar drainage of cerebrospinal fluid after subarachnoid hemorrhage. J. Neurosurg. 100 215-224 (2004)

165. Kwon, O. Y., Kim, Y. J., Cho, C. S., Lee, S. K. \& Cho, M. K. The utility and benefits of external lumbar CSF drainage after endovascular coiling on aneurysmal subarachnoid hemorrhage. J. Korean Neurosurg. Soc. 43, 281-287 (2008).

166. Sun, C. et al. Choice for the removal of bloody cerebrospinal fluid in postcoiling aneurysmal subarachnoid hemorrhage: external ventricular drainage or lumbar drainage? Turk. Neurosurg. 24 737-744 (2014)

167. Al-Tamimi, Y. Z. et al. Lumbar drainage of cerebrospinal fluid after aneurysmal subarachnoid hemorrhage: a prospective, randomized, controlled trial (LUMAS). Stroke 43, 677-682 (2012). This article presents the first definitive trial of CSF drainage after SAH. 
168. Bardutzky, J. et al. EARLYDRAIN- outcome after early lumbar CSF-drainage in aneurysmal subarachnoid hemorrhage: study protocol for a randomized controlled trial. Trials 12, 203 (2011).

169. Mendelow, A. D. et al. Early surgery versus initial conservative treatment in patients with spontaneous supratentorial intracerebral haematomas in the International Surgical Trial in Intracerebral Haemorrhage (STICH): a randomised trial. Lancet 365, 387-397 (2005).

170. Mendelow, A. D. et al. Early surgery versus initial conservative treatment in patients with spontaneous supratentorial lobar intracerebral haematomas (STICH II): a randomised trial. Lancet 382, 397-408 (2013)

171. Mould, W. A. et al. Minimally invasive surgery plus recombinant tissue-type plasminogen activator for intracerebral hemorrhage evacuation decreases perihematomal edema. Stroke 44, 627-634 (2013)

172. US National Library of Medicine. ClinicalTrials.gov https://www.clinicaltrials.gov/ct2/show/NCT01827046 (2017).

173. Nonaka, T., Watanabe, S., Chigasaki, H., Miyaoka, M. \& Ishii, S. Etiology and treatment of vasospasm following subarachnoid hemorrhage. Neurol. Med. Chir 19, 53-60 (1979).

174. Shindo, S. Haptoglobin subtyping with anti-haptoglobin alpha chain antibodies. Electrophoresis 11, 483-488 (1990).

175. Lipiski, M. et al. Human Hp1-1 and Hp2-2 phenotype specific haptoglobin therapeutics are both effective in vitro and in guinea pigs to attenuate hemoglobin toxicity. Antioxid. Redox Signal. 19, 1619-1633 (2013).

176. Kubota, K., Egi, M. \& Mizobuchi, S. Haptoglobin administration in cardiovascular surgery patients: its association with the risk of postoperative acute kidney injury. Anesth. Analg. 124, 1771-1776 (2017).

177. Muta, T. et al. Anti-haptoglobin antibody detection after febrile non-hemolytic transfusion reactions in a non-haptoglobin-deficient patient. Transfus. Apher. Sci. 41, 171-173 (2009).

178. Chen, G., Fang, Q., Zhang, J., Zhou, D. \& Wang, Z Role of the Nrf2-ARE pathway in early brain injury after experimental subarachnoid hemorrhage. J. Neurosci. Res. 89, 515-523 (2011). This study is the first demonstration of the potential therapeutic role of NRF2 upregulation after SAH.

179. Zhao, X. D., Zhou, Y. T. \& Lu, X. J. Sulforaphane enhances the activity of the Nrf2-ARE pathway and attenuates inflammation in $\mathrm{OxyHb}$-induced rat vascular smooth muscle cells. Inflamm. Res. 62 857-863 (2013).

180. Zhao, X., Wen, L., Dong, M. \& Lu, X. Sulforaphane activates the cerebral vascular Nrf2-ARE pathway and suppresses inflammation to attenuate cerebral vasospasm in rat with subarachnoid hemorrhage. Brain Res. 1653, 1-7 (2016)

181. US National Library of Medicine. ClinicalTrials.gov https://www.clinicaltrials.gov/ct2/show/NCT02614742 (2018).

182. Zhao, X. R., Gonzales, N. \& Aronowski, J. Pleiotropic role of PPAR $\gamma$ in intracerebral hemorrhage: an intricate system involving Nrf2, RXR, and NF-kB. CNS Neurosci. Ther. 21, 357-366 (2015)

183. Zhao, X. et al. Hematoma resolution as a target for intracerebral hemorrhage treatment: role for peroxisome proliferator-activated receptor gamma in microglia/macrophages. Ann. Neurol. 61, 352-362 (2007).

184. Lin, B. F. et al. Rosiglitazone attenuates cerebra vasospasm and provides neuroprotection in an experimental rat model of subarachnoid hemorrhage. Neurocrit Care. 21, 316-331 (2014).

185. Gonzales, N. R. et al. Design of a prospective, doseescalation study evaluating the Safety of Pioglitazone for Hematoma Resolution in Intracerebral Hemorrhage (SHRINC). Int. J. Stroke. 8, 388-396 (2013).

186. Cho, H. Y. et al. Nrf2-regulated PPAR \{gamma\} expression is critical to protection against acute lung injury in mice. Am. J. Respir. Crit. Care Med. 182. 170-182 (2010)

187. Park, E. Y., Cho, I. J. \& Kim, S. G. Transactivation of the PPAR-responsive enhancer module in chemopreventive glutathione S-transferase gene by the peroxisome proliferator-activated receptor-gamma and retinoid $\mathrm{X}$ receptor heterodimer. Cancer Res. 64, 3701-3713 (2004)

188. Selim, M. et al. Safety and tolerability of deferoxamine mesylate in patients with acute intracerebral hemorrhage. Stroke 42, 3067-3074 (2011).
189. Yeatts, S. D., Palesch, Y. Y., Moy, C. S. \& Selim, M. High dose deferoxamine in intracerebral hemorrhage (HI-DEF) trial: rationale, design, and methods. Neurocrit Care. 19, 257-266 (2013)

190. US National Library of Medicine. ClinicalTrials.gov https://www.clinicaltrials.gov/ct2/show/NCT02175225 (2018).

191. Yu, Y. et al. The clinical effect of deferoxamine mesylate on edema after intracerebral hemorrhage. PLOS ONE. 10, e0122371 (2015).

192. US National Library of Medicine. ClinicalTrials.gov https://www.clinicaltrials.gov/ct2/show/NCT02875262 (2017).

193. Levy, M. \& Llinas, R. Pilot safety trial of deferiprone in 10 subjects with superficial siderosis. Stroke 43, 120-124 (2012)

194. Kessler, R. A. et al. Two-year observational study of deferiprone in superficial siderosis. CNS Neurosci. Ther 24, 187-192 (2017)

195. World Health Organization. International Clinical Trials Registry Platform. WHO http://apps.who.int/ trialsearch/Trial3. aspx?trialid=JPRNUMIN000021218 (2016)

196. Zhang, S., Wang, L., Liu, M. \& Wu, B. Tirilazad for aneurysmal subarachnoid haemorrhage. Cochrane Database Syst. Rev. 17, CD006778 (2010).

197. Galea, J et al. Intravenous anakinra can achieve experimentally effective concentrations in the central nervous system within a therapeutic time window: results of a dose-ranging study. J. Cereb. Blood Flow Metab. 31, 439-447 (2011)

198. Galea, J. et al. Reduction of inflammation after administration of interleukin-1 receptor antagonist following aneurysmal subarachnoid hemorrhage: results of the Subcutaneous Interleukin-1 Ra in SAH (SCIL-SAH) study. J. Neurosurg. 128, 515-523 (2017).

199. US National Library of Medicine. ClinicalTrials.gov https://www.clinicaltrials.gov/ct2/show/NCT03249207 (2017).

200. Oldfield, E. H. et al. Safety and pharmacokinetics of sodium nitrite in patients with subarachnoid hemorrhage: a phase lla study. J. Neurosurg. 119, 634-641 (2013)

201. US National Library of Medicine. ClinicalTrials.gov https://www.clinicaltrials.gov/ct2/show/NCT02176837 (2018).

202. Bath, P. M. et al. Efficacy of nitric oxide, with or without continuing antihypertensive treatment, for management of high blood pressure in acute stroke (ENOS): a partial-factorial randomised controlled trial. Lancet 385, 617-628 (2015).

203. Bjornsson, E. et al. A rare splice donor mutation in the haptoglobin gene associates with blood lipid levels and coronary artery disease. Hum. Mol. Genet. 26, 2364-2376 (2017).

204. Melamed-Frank, M. et al. Structure-function analysis of the antioxidant properties of haptoglobin. Blood 98, 3693-3698 (2001)

205. Asleh, R., Guetta, J., Kalet-Litman, S., Miller-Lotan, R. $\&$ Levy, A. P. Haptoglobin genotype- and diabetesdependent differences in iron-mediated oxidative stress in vitro and in vivo. Circ. Res. 96, 435-441 (2005).

206. Bamm, V. V., Tsemakhovich, V. A., Shaklai, M. \& Shaklai, N. Haptoglobin phenotypes differ in their ability to inhibit heme transfer from hemoglobin to LDL. Biochemistry 43, 3899-3906 (2004).

207. Pimenova, T. et al. Quantitative mass spectrometry defines an oxidative hotspot in hemoglobin that is specifically protected by haptoglobin. J. Proteome Res. 9, 4061-4070 (2010).

208. Mollan, T. L. et al. Redox properties of human hemoglobin in complex with fractionated dimeric and polymeric human haptoglobin. Free Radic. Biol. Med. 69, 265-277 (2014).

209. Asleh, R. et al. Genetically determined heterogeneity in hemoglobin scavenging and susceptibility to diabetic cardiovascular disease. Circ. Res. 92 1193-1200 (2003).

210. Na, N., Ouyang, J., Taes, Y. E. \& Delanghe, J. R. Serum free hemoglobin concentrations in healthy individuals are related to haptoglobin type. Clin. Chem. $\mathbf{5 1}$ 1754-1755 (2005).

211. Guetta, J., Strauss, M., Levy, N. S., Fahoum, L. \& Levy, A. P. Haptoglobin genotype modulates the balance of Th $1 /$ Th2 cytokines produced by macrophages exposed to free hemoglobin. Atherosclerosis 191, 48-53 (2007).

212. Bechmann, I., Galea, I. \& Perry, V. H. What is the blood-brain barrier (not)? Trends Immunol. 28, 5-11 (2007).
213. Thompson, E. J. in Proteins of the Cerebrospinal Fluid: Analysis \& Interpretation in the Diagnosis and Treatment of Neurological Disease (ed. Thompson, E. J.) 13-30 (Elsevier, 2005).

214. Andersen, C. B. F. et al. Haptoglobin. Antioxid. Redox Signal. 26, 814-831 (2017).

215. Barua, N. U. et al. Intrastriatal convection-enhanced delivery results in widespread perivascular distribution in a pre-clinical model. Fluids Barriers CNS. 9, 2 (2012)

216. Carare, R. O. et al. Solutes, but not cells, drain from the brain parenchyma along basement membranes of capillaries and arteries: significance for cerebral amyloid angiopathy and neuroimmunology. Neuropathol. Appl. Neurobiol. 34, 131-144 (2008).

217. Iliff, J. J. et al. Brain-wide pathway for waste clearance captured by contrast-enhanced MRI. J. Clin. Invest. 123, 1299-1309 (2013)

218. Larsson, M., Cheng, T.-M., Chen, C.-Y. \& Mao, S. J. T. in Acute Phase Proteins (ed. Janciauskiene, S.) 163-180 (InTech, 2013).

219. Mosconi, E. et al. Breast milk immune complexes are potent inducers of oral tolerance in neonates and prevent asthma development. Mucosal Immunol. 3 461-474 (2010)

220. Carare, R. O. et al. Immune complex formation impairs the elimination of solutes from the brain implications for immunotherapy in Alzheimer's disease. Acta Neuropathol. Commun. 1, 48 (2013).

221. Galea, I., Bechmann, I. \& Perry, V. H. What is immune privilege (not)? Trends Immunol. 28, 12-18 (2007).

222. Andersson, P. B., Perry, V. H. \& Gordon, S. The acute inflammatory response to lipopolysaccharide in CNS parenchyma differs from that in other body tissues. Neuroscience 48, 169-186 (1992).

223. Engelhardt, B. et al. Vascular, glial, and lymphatic immune gateways of the central nervous system. Acta Neuropathol. 132, 317-338 (2016).

224. US National Library of Medicine. ClinicalTrials.gov https://www.clinicaltrials.gov/ct2/show/NCT03040128 (2017).

225. US National Library of Medicine. ClinicalTrials.gov https://www.clinicaltrials.gov/ct2/show/NCT01805895 (2018).

226. US National Library of Medicine. ClinicalTrials.gov http://www.clinicaltrials.gov/ct2/show/NCT02113176 (2014).

227. World Health Organization. International Clinical Trials Registry Platform. WHO http://apps.who.int/ trialsearch/Trial2.aspx?TriallD=EUCTR2005-00468431-GB (2012)

228. US National Library of Medicine. ClinicalTrials.gov https://www.clinicaltrials.gov/ct2/show/NCT00585559 (2018).

229. Shao, A. et al. Hydrogen-rich saline attenuated subarachnoid hemorrhage-induced early brain injury in rats by suppressing inflammatory response: possible involvement of NF-kB pathway and NLRP3 inflammasome. Mol. Neurobiol. 53, 3462-3476 (2016).

230. World Health Organization. International Clinical Trials Registry Platform. WHO http://apps.who.int/ trialsearch/Trial2.aspx?TrialID=ChiCTR-IIR-16009220 (2017).

231. Lyden, P. D. et al. Safety and tolerability of NXY-059 for acute intracerebral hemorrhage: the CHANT Trial. Stroke 38, 2262-2269 (2007).

232. Dixon, S. J. et al. Ferroptosis: an iron-dependent form of nonapoptotic cell death. Cell 149, 1060-1072 (2012).

233. Li, Q. et al. Inhibition of neuronal ferroptosis protects hemorrhagic brain. JCI Insight 2, e90777 (2017)

234. Liu, Y. et al. The 5-lipoxygenase inhibitor zileuton confers neuroprotection against glutamate oxidative damage by inhibiting ferroptosis. Biol. Pharm. Bull. 38, 1234-1239 (2015).

235. Zilka, O. et al. On the mechanism of cytoprotection by ferrostatin-1 and liproxstatin- 1 and the role of lipid peroxidation in ferroptotic cell death. ACS Cent Sci. 3 232-243 (2017)

236. King, M. D., Whitaker-Lea, W. A., Campbell, J. M., Alleyne, C. H. \& Dhandapani, K. M. Necrostatinreduces neurovascular injury after intracerebral hemorrhage. Int. J. Cell Biol. 2014, 495817 (2014).

237. Rushmore, T. H., Morton, M. R. \& Pickett, C. B. The antioxidant responsive element. Activation by oxidative stress and identification of the DNA consensus sequence required for functional activity. J. Biol. Chem. 266, 11632-11639 (1991).

238. Sandberg, M., Patil, J., D’Angelo, B., Weber, S. G. \& Mallard, C. NRF2-regulation in brain health and disease: implication of cerebral inflammation. Neuropharmacology 79, 298-306 (2014). 


\section{REVIEWS}

\section{Acknowledgements}

The authors thank the University of Southampton Global Partnership Award Scheme and NeuroSpring for their support. NeuroSpring is a nonprofit organization with the primary aim of supporting research in the neurosciences leading to new medical therapies (http://www. neurospring. org/); one of the authors (M.B.) is a senior scientist at NeuroSpring.

\section{Author contributions}

I.G. led the Review with D.B. and B.G. Each author wrote a review of a subtopic. These subtopical reviews were then integrated by D.B., B.G. and I.G. into a unified first draft, which was further revised by all authors. Figures were prepared by A.Z., J.G. and I.G. and were reviewed by all authors.

\section{Competing interests}

The authors declare no competing interests.

\section{Publisher's note}

Springer Nature remains neutral with regard to jurisdictional claims in published maps and institutional affiliations.

\section{Review criteria}

PubMed was searched for articles published in English prior to June 2017 using the following search terms: "h(a)emoglobin",

"meth(a)emoglobin", "h(a)eme", "h(a)emolysis", "haptoglobin", "CD163", "h(a)emopexin", "CD91 OR LRP1", "HO-1 OR HO1 OR HO-2 OR HO2 OR h(a)eme oxygenase", "bilirubin", "iron", "ferritin" and "h(a)emosiderin" in single combinations with "neurological", "brain", "intracerebral", "intracranial", "subarachnoid", "h(a)emorrhage", "treatment", "trial", "toxic(ity)", "outcome(s)", and "gene(tics)". Clinical trial registries (European Clinical Trials Database, ClinicalTrials.gov, the International Standard Registered Clinical/Social Study Number (ISRCTN) registry and the WHO International Clinical Trials Registry Platform) were searched for treatments in subarachnoid haemorrhage, intracerebral haemorrhage and superficial siderosis. The reference lists of published articles were manually searched for further articles. All clinical studies reviewed were assessed for quality by D.B., B.G. and I.G. by tabulating study design, sample size, risk of bias and study quality using the Cochrane Tool (for trials) or the Newcastle-Ottawa Score (for observational studies), Cohen's effect size and $P$ value (Supplementary Tables 1,2).

\section{Supplementary information}

Supplementary information is available for this paper at https://doi.org/10.1038/s41582-018-0020-0. 